\title{
Quartic Rational Said-Ball-Like Basis with Tension Shape Parameters and Its Application
}

\author{
Yuanpeng Zhu, Xuli Han, and Shengjun Liu \\ School of Mathematics and Statistics, Central South University, Changsha 410083, China \\ Correspondence should be addressed to Shengjun Liu; shjliu.cg@csu.edu.cn
}

Received 22 March 2014; Accepted 5 June 2014; Published 26 June 2014

Academic Editor: Fernando Simões

Copyright (C) 2014 Yuanpeng Zhu et al. This is an open access article distributed under the Creative Commons Attribution License, which permits unrestricted use, distribution, and reproduction in any medium, provided the original work is properly cited.

\begin{abstract}
Four new quartic rational Said-Ball-like basis functions, which include the cubic Said-Ball basis functions as a special case, are constructed in this paper. The new basis is applied to generate a class of $C^{1}$ continuous quartic rational Hermite interpolation splines with local tension shape parameters. The error estimate expression of the proposed interpolant is given and the sufficient conditions are derived for constructing a $C^{1}$ positivity- or monotonicity- preserving interpolation spline. In addition, we extend the quartic rational Said-Ball-like basis to a triangular domain which has three tension shape parameters and includes the cubic triangular Said-Ball basis as a special case. In order to compute the corresponding patch stably and efficiently, a new de Casteljautype algorithm is developed. Moreover, the $G^{1}$ continuous conditions are deduced for the joining of two patches.
\end{abstract}

\section{Introduction}

Constructing practical basis functions to generate free form curves and surfaces is an important topic of CAGD (computer aided geometric design) and computer graphics. Generally speaking, the basis function with good properties plays a vital role in curve and surface design. In [1-3], Ball introduced a kind of cubic rational polynomial to be the basis of CONSURF surface lofting program. In [4], Said extended the cubic basis proposed by Ball into arbitrary odd degree basis to get a type of generalized Ball basis. After that, Goodman and Said proved that the generalized Ball basis is normalized totally positive and hence it possesses the same kind of shape preserving properties as the Bernstein basis [5]. In [6], Hu and his colleagues suggested an extension of the generalized Ball basis to arbitrary even degree, usually known as Said-Ball basis. In [7], based on the necessary and sufficient conditions for the conic representation in a rational low degree Bézier form and the transformation formula from Bernstein basis to Said-Ball basis, Hu and Wang deduced the representation theory of rational cubic and quartic Said-Ball conics. Because surfaces modeling over a triangular domain have great potential of constructing complex shapes, there are some researchers who have put many efforts on the establishments of triangular Said-Ball bases. In [8], Goodman and Said defined a bivariate Said-Ball basis on a triangle and designed a triangular Said-Ball surface based on the research of Said-Ball curve. In [9], Hu et al. extended the univariate Wang-Ball basis to the bivariate case on a triangle, designed a triangular Wang-Ball surface, and pointed out its virtues. Chen and Wang [10] constructed a class of triangular DP surfaces. Recently, Zhu and Han [11] constructed a class of $\alpha \beta \gamma$-Bernstein-Bézier basis possessing three-exponentialshape parameters over a triangular domain, which includes the cubic triangular Said-Ball basis and the cubic triangular Bernstein-Bézier basis as special cases.

In industrial design and scientific data visualization, constructing shape preserving interpolation splines for the interpolation of positive or monotonic data is an essential problem and has attracted widespread interest. In [12], Schmidt and Heß derived a necessary and sufficient criterion under which a kind of $C^{1}$ quadratic rational positivitypreserving interpolation spline was developed. Sakai and Schmidt [13] proposed a method for the construction of positive cubic rational splines of continuity class $C^{2}$. In $[14,15]$, two kinds of $C^{1}$ rational cubic interpolation splines 
were constructed by Hussain and Sarfraz to visualize the positive data. In [16], Fritsch and Carlson gave necessary and sufficient conditions for a cubic spline to be monotone on an interval. Manni and Sablonnière [17] developed an explicit expression of a $C^{1}$ cubic rational spline which can be applied to monotonic data. In $[18,19]$, two kinds of $C^{2}$ cubic rational interpolation splines were proposed by Sarfraz for the visualization of monotonic data. In [20], Hussain and Sarfraz presented a $C^{1}$ cubic rational interpolating scheme to deal with the problem of monotonicity. In [21], by using weighted $C^{1}$ quadratic splines, Kvasov proposed two algorithms with automatic selection of the shape parameters to construct a shape preserving interpolation spline for monotonic or convex data. Recently, the quadratic and cubic rational trigonometric bases are also considered by some scholars to construct positivity-preserving and monotonicity-preserving interpolation splines; see [22-24].

The purpose of this paper is to present four new quartic rational Said-Ball-like basis functions with two-tensionshape parameters, which include the cubic Said-Ball basis functions. By using the new basis, a class of $C^{1}$ continuous quartic rational Hermite interpolation spline with local tension shape parameters is also constructed. Its error estimate expression is given and sufficient conditions for constructing a $C^{1}$ positivity- or monotonicity-preserving interpolation spline are derived. The quartic rational Said-Ball-like basis functions are also extended to a triangular domain. In order to compute the corresponding patch stably and efficiently, a new de Casteljau-type algorithm is developed.

The rest of this paper is organized as follows. Section 2 gives the definition and properties of the new quartic rational Said-Ball-like basis and discusses the properties of the corresponding quartic rational Said-Ball-like curve. In Section 3, based on the new proposed quartic rational SaidBall-like basis, a new class of $C^{1}$ quartic rational Hermite interpolation spline is developed. Sufficient conditions are derived for constructing a $C^{1}$ positivity- or monotonicitypreserving interpolation spline. Section 4 gives the construction and properties of the quartic rational Said-Ball-like basis functions over a triangular domain. The conditions for $G^{1}$ continuously and smoothly joining of two quartic rational triangular Said-Ball-like patches are deduced. Conclusion is given in Section 5.

\section{Quartic Rational Said-Ball-Like Basis}

2.1. Construction of the Basis. We construct four new quartic rational Said-Ball-like basis functions as follows.

Definition 1. For any $\alpha, \beta \in[0,+\infty), t \in[0,1]$, the new quartic rational Said-Ball-like basis functions are given by

$$
\begin{aligned}
& B_{0}(t)=\frac{(1-t)^{2}}{1+\alpha t}, \\
& B_{1}(t)=\frac{(1-t)^{2} t}{1+\alpha t}(2+\alpha+2 \alpha t),
\end{aligned}
$$

$$
\begin{aligned}
& B_{2}(t)=\frac{(1-t) t^{2}}{1+\beta(1-t)}[2+\beta+2 \beta(1-t)], \\
& B_{3}(t)=\frac{t^{2}}{1+\beta(1-t)} .
\end{aligned}
$$

Notice that

$$
\begin{aligned}
& B_{1}(t)=1-3 t^{2}+2 t^{3}-B_{0}(t), \\
& B_{2}(t)=3 t^{2}+2 t^{3}-B_{3}(t) ;
\end{aligned}
$$

we can see immediately that the quartic rational Said-Balllike basis functions (1) are constructed in the function space $\operatorname{span}\left\{1,3 t^{2}-2 t^{3},(1-t)^{2} /(1+\alpha t), t^{2} /[1+\beta(1-t)]\right\}$. It is easy to check that, for $\alpha=\beta=0$, the quartic rational Said-Ball-like basis is precisely the cubic Said-Ball basis; see [1-3].

For convenience, in the following discussion we will also denote the four quartic rational Said-Ball-like basis functions as $B_{i}(t ; \alpha, \beta), i=0,1,2,3$, or $B_{i}(t ; \alpha), i=0,1$, and $B_{i}(t ; \beta)$, $i=3,4$. Figure 1 shows some plots of the new basis functions for some values of the shape parameters.

From the definition of the quartic rational Said-Ball-like basis given in (1), we have the following important properties of the new basis.

Theorem 2. The quartic rational Said-Ball-like basis (1) has the following properties.

(a) Partition of unity: $\sum_{i=0}^{3} B_{i}(t ; \alpha, \beta)=1$.

(b) Nonnegativity: $B_{i}(t ; \alpha, \beta) \geq 0, i=0,1,2,3$.

(c) Symmetry: $B_{i}(t ; \alpha, \beta)=B_{3-i}(1-t ; \beta, \alpha)$, for $i=0,1$.

(d) Linear independence: for $\alpha, \beta \in(2,+\infty)$, the quartic rational Said-Ball-like basis functions $B_{i}(t ; \alpha, \beta), i=$ $0,1,2,3$, are linearly independent.

Proof. It is easy to check that the basis (1) has the properties of partition of unity, nonnegativity, and symmetry. In addition, for any $\alpha, \beta \in[0,+\infty), \xi_{i} \in \mathbb{R}(i=0,1,2,3)$, we consider a linear combination

$$
\sum_{i=0}^{3} \xi_{i} B_{i}(t ; \alpha, \beta)=0 .
$$

Differentiating with respect to the variable $t$ on both sides, we have

$$
\sum_{i=0}^{3} \xi_{i} B_{i}^{\prime}(t ; \alpha, \beta)=0 .
$$

For $t=0$, from (3) and (4), we can obtain the following system of equations with respect to $\xi_{0}$ and $\xi_{1}$ :

$$
\begin{gathered}
\xi_{0}=0, \\
(2+\alpha)\left(\xi_{1}-\xi_{0}\right)=0 .
\end{gathered}
$$

Then, we have $\xi_{0}=\xi_{1}=0$. Similarly, for $t=1$, we can get $\xi_{2}=$ $\xi_{3}=0$. Thus, the basis functions (1) are linearly independent. 


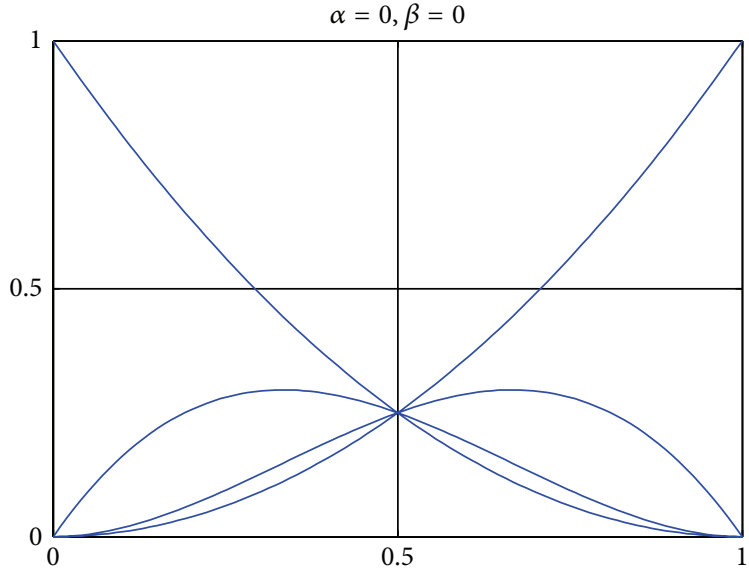

(a)

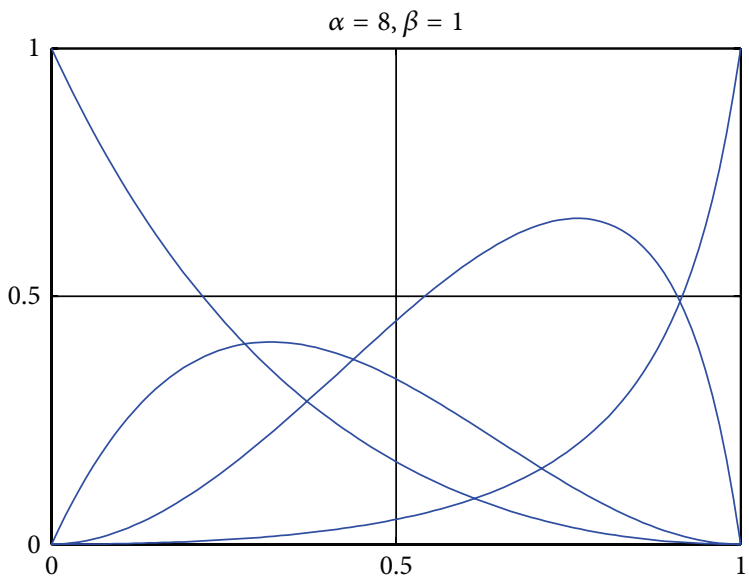

(c)

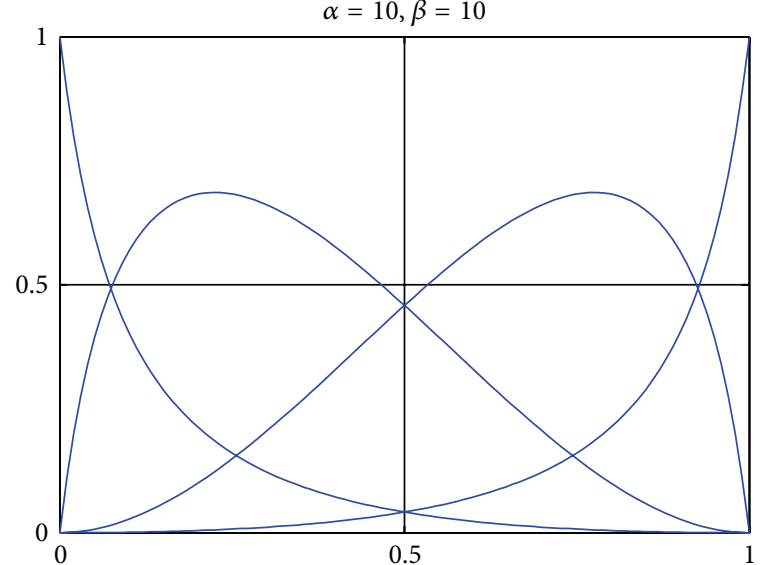

(b)

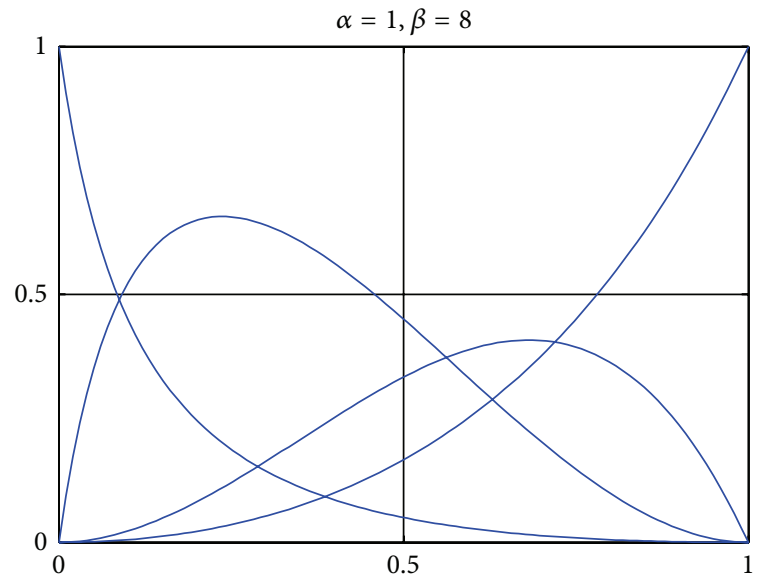

(d)

Figure 1: Some plots of the new quartic rational Said-Ball-like basis functions.

\subsection{Definition and Properties of the Quartic Rational Said-}

\section{Ball-Like Curve}

Definition 3. Given control points $Q_{i}(i=0,1,2,3)$ in $\mathbb{R}^{2}$ or $\mathbb{R}^{3}$, then

$$
R(t ; \alpha, \beta)=\sum_{i=0}^{3} B_{i}(t ; \alpha, \beta) Q_{i}, \quad t \in[0,1], \alpha, \beta \in[0,+\infty)
$$

is called a quartic rational Said-Ball-like curve with two shape parameters $\alpha$ and $\beta$.

Since the quartic rational Said-Ball-like basis (1) has the properties of partition of unity and nonnegativity, we can see that the corresponding curve (6) defined by it has the properties of affine invariance and convex hull, which are crucial properties in curve design. Direct computation gives the following end-point property of the quartic rational SaidBall-like curve:

$$
\begin{aligned}
& R(0 ; \alpha, \beta)=Q_{0}, \\
& R(1 ; \alpha, \beta)=Q_{3},
\end{aligned}
$$

$$
\begin{aligned}
& R^{\prime}(0 ; \alpha, \beta)=(2+\alpha)\left(Q_{1}-Q_{0}\right), \\
& R^{\prime}(1 ; \alpha, \beta)=(2+\beta)\left(Q_{3}-Q_{2}\right) .
\end{aligned}
$$

These imply that for any $\alpha, \beta \in[0,+\infty)$ the curve has the end-point interpolation property and $Q_{0} Q_{1}, Q_{2} Q_{3}$ are the tangent lines of the curve at the points $Q_{0}, Q_{3}$, respectively. From these, we can find that the quartic rational Said-Balllike curve has some properties analogous to that of the cubic Said-Ball curve. For $\alpha=\beta=0$, the quartic rational Said-Balllike curve, specially, is just the cubic Said-Ball curve.

For the analysis of the effect of the shape parameters on the shape of the obtained quartic rational Said-Ball-like curve, we rewrite (6) as follows:

$$
\begin{aligned}
R(t ; \alpha, \beta)= & (1-t)^{2}(1+2 t) Q_{1}+t^{2}(3-2 t) Q_{2} \\
& +B_{0}(t ; \alpha)\left(Q_{0}-Q_{1}\right)+B_{3}(t ; \beta)\left(Q_{3}-Q_{2}\right) .
\end{aligned}
$$

From (8), it is obvious that the shape parameters $\alpha$ and $\beta$ only affect the curve on the control edges $Q_{0}-Q_{1}$ and 


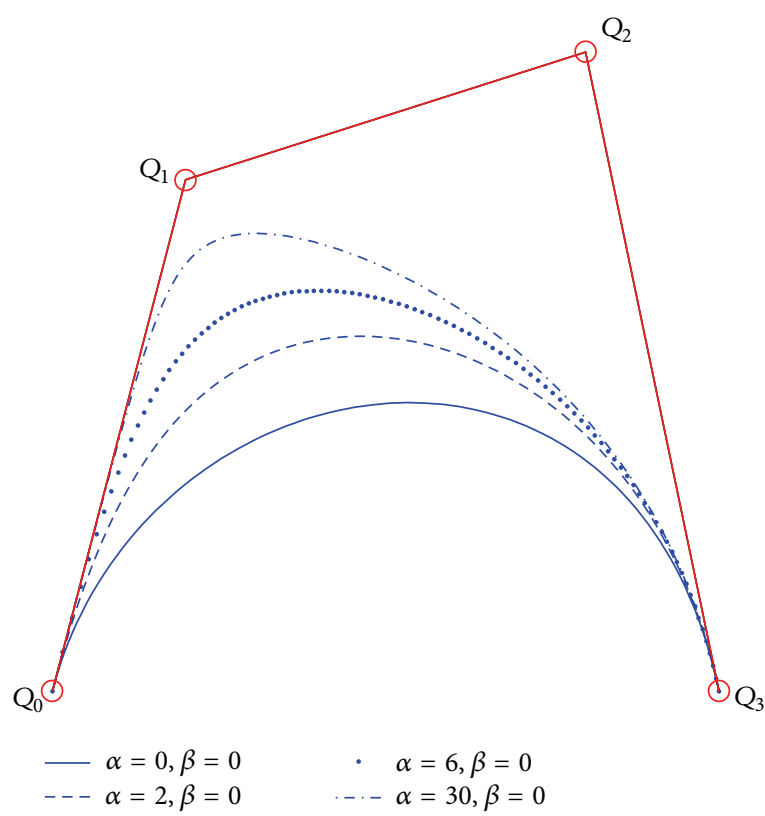

(a)

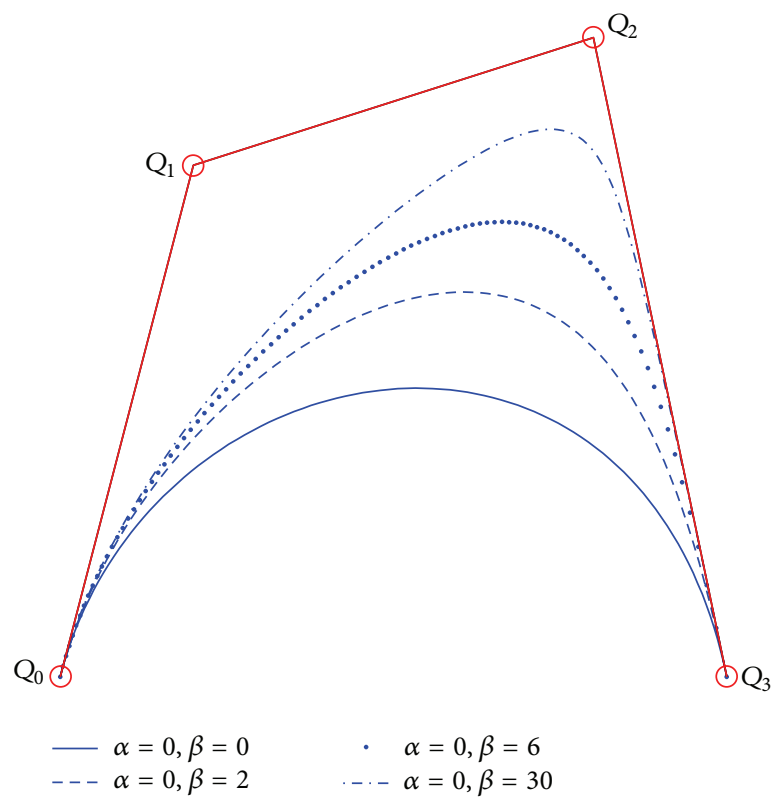

(b)

FIGURE 2: The effect of shape parameters on the quartic rational Said-Ball-like curves.

$Q_{3}-Q_{2}$, respectively. Moreover, $B_{0}(t ; \alpha)$ decreases with the increase of $\alpha$ for any fixed $t \in(0,1)$, which indicates that the curve moves in the same direction of the edge $Q_{0}-Q_{1}$ as $\alpha$ increases. Inversely, as $\alpha$ decreases, the curve moves in the opposite direction to the edge $Q_{0}-Q_{1}$. The shape parameter $\beta$ has similar effects on the edge $Q_{3}-Q_{2}$. When the shape parameters satisfy $\alpha=\beta$, the curve moves in the same direction or the opposite direction to the edge $Q_{2}-Q_{1}$ when $\alpha$ increases or decreases, respectively. And when $\alpha, \beta$ increase at the same time, the curve tends to the edge $Q_{2}-Q_{1}$. Thus we can see that the shape parameters $\alpha$ and $\beta$ serve as local tension parameters. Figure 2 shows the effect of the shape parameters on the quartic rational Said-Ball-like curves.

Corner cutting algorithm is an efficient and stable process for computing the quartic rational Said-Ball-like curve. In order to develop such an algorithm, we rewrite the quartic rational Said-Ball-like curve (6) in the following matrix form:

$$
\begin{aligned}
R(t ; \alpha, \beta) & \\
= & (1-t \quad t)\left(\begin{array}{cccc}
\frac{1}{1+\alpha t} & \frac{\alpha t}{1+\alpha t} & 0 & 0 \\
0 & 1-t & t
\end{array}\right) \\
& \times\left(\begin{array}{cccc}
1+t & & 0 \\
0 & 1-t & 1 \\
0 & 0 & \frac{\beta(1-t)}{1+\beta(1-t)} & 1+\beta(1-t)
\end{array}\right) \\
& \times\left(\begin{array}{l}
Q_{0} \\
Q_{1} \\
Q_{2} \\
Q_{3}
\end{array}\right) .
\end{aligned}
$$

From (9), we can immediately obtain a corner cutting algorithm for computing the quartic rational Said-Ball-like curve. See Figure 3 for an illustration of this algorithm.

Control polygons provide an important tool in geometric modeling. Here, we adopt the method given in [25] to show some relations of the quartic rational Said-Ball-like curves and the classical cubic Bézier curves corresponding to the same control polygons.

Suppose that the control points $Q_{i}(i=0,1,2,3)$ are not collinear; let $A(t)$ be a cubic Bézier curve $A(t)=$ $\sum_{i=0}^{3} Q_{i}\left(\begin{array}{c}3 \\ i\end{array}\right)(1-t)^{3-i} t$, where $t \in[0,1]$ and $R(t ; \alpha, \beta)$ with the same control points $Q_{i}(i=0,1,2,3)$. Then, it is easy to show that $R(0 ; \alpha, \beta)=A(0), R(1 ; \alpha, \beta)=A(1)$. Moreover, according to a simple computation, we have

$$
R\left(\frac{1}{2} ; \alpha, \alpha\right)-Q^{*}=f(\alpha)\left[A\left(\frac{1}{2}\right)-Q^{*}\right]
$$

where $Q^{*}=(1 / 2)\left(P_{1}+P_{2}\right), f(\alpha)=4 /(2+\alpha)$.

From (10), we can see that if $f(\alpha)<1$, that is, $\alpha>2$, then the quartic rational Said-Ball-like curves $R(t ; \alpha, \alpha)$ will be closer to the control polygon than the cubic Bézier curve $A(t)$.

Figure 4 shows the comparisons between the quartic rational Said-Ball-like curves and the cubic Bézier curves. It is clear that, by changing the shape parameters, the quartic rational Said-Ball-like curves can yield tight envelopes for the cubic Bézier curves. Figure 5 shows the comparisons between the quartic rational Said-Ball-like curves (solid lines and dash-dotted lines) and the cubic trigonometric Bézier curves with two shape parameters $\lambda$ and $\mu$ given in [25] (dashed lines and dotted lines). Clearly, the quartic rational Said-Ball-like curves can be closer to the control polygon than the cubic trigonometric Bézier curves. 


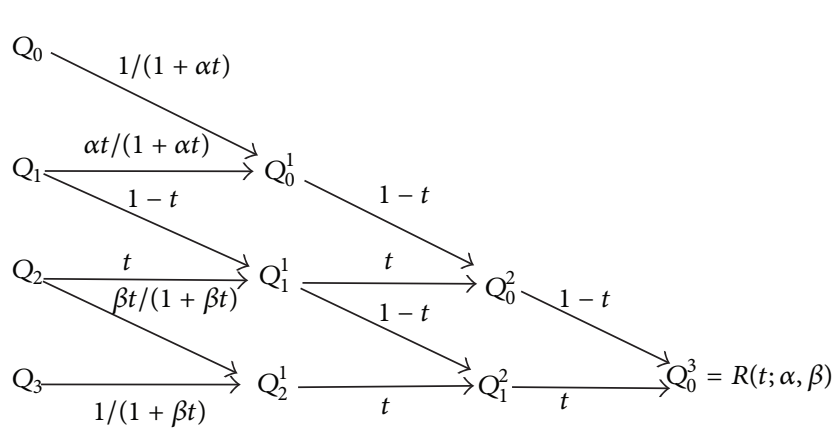

(a)

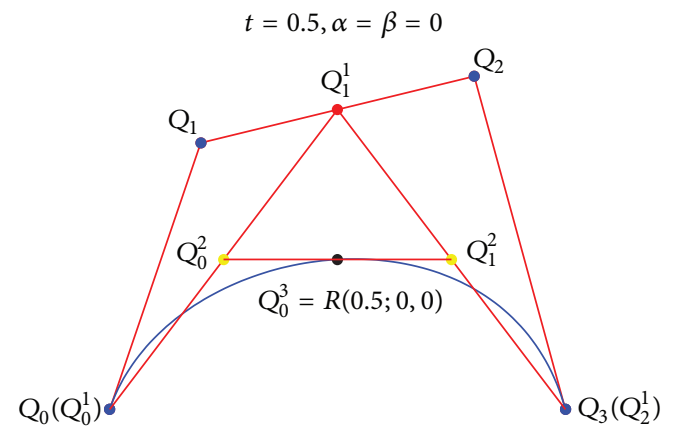

(b)

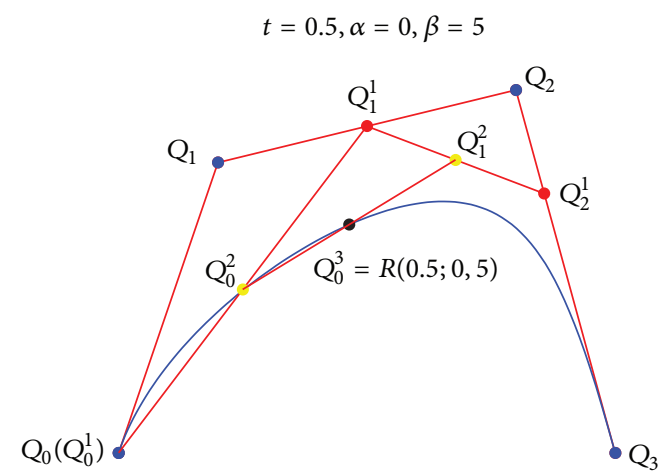

(c)

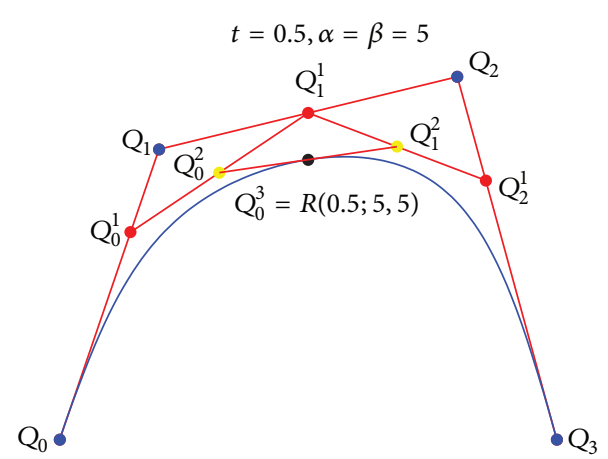

(d)

FIgURE 3: Corner cutting algorithm.

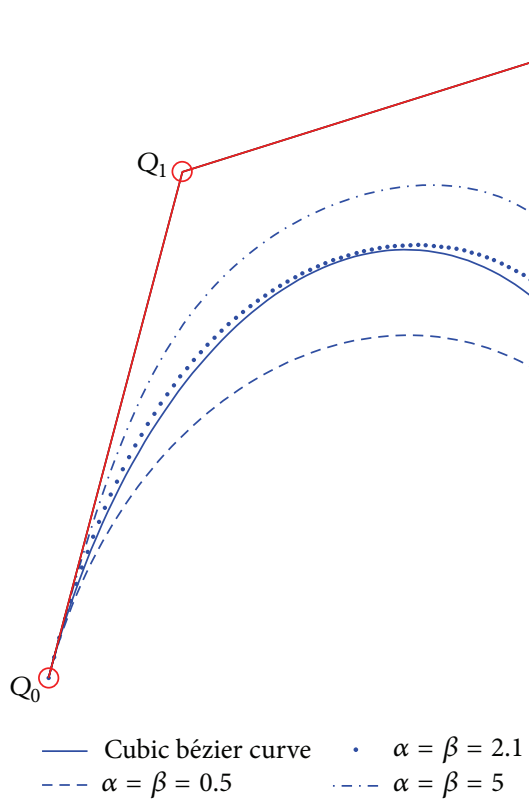

(a)

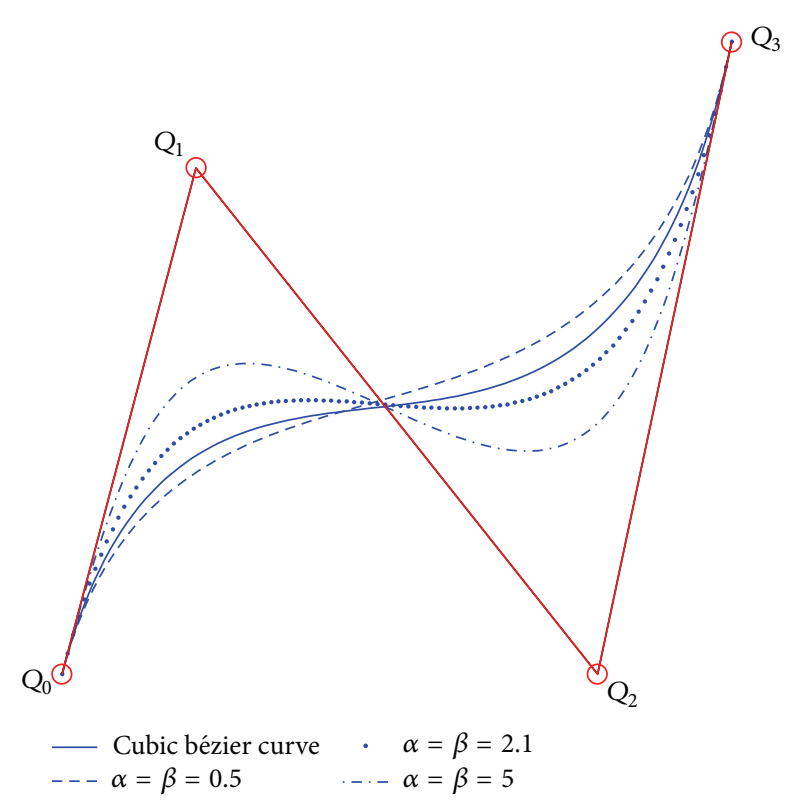

(b)

FIgURE 4: Comparisons between the quartic rational Said-Ball-like curves and the cubic Bézier curves. 


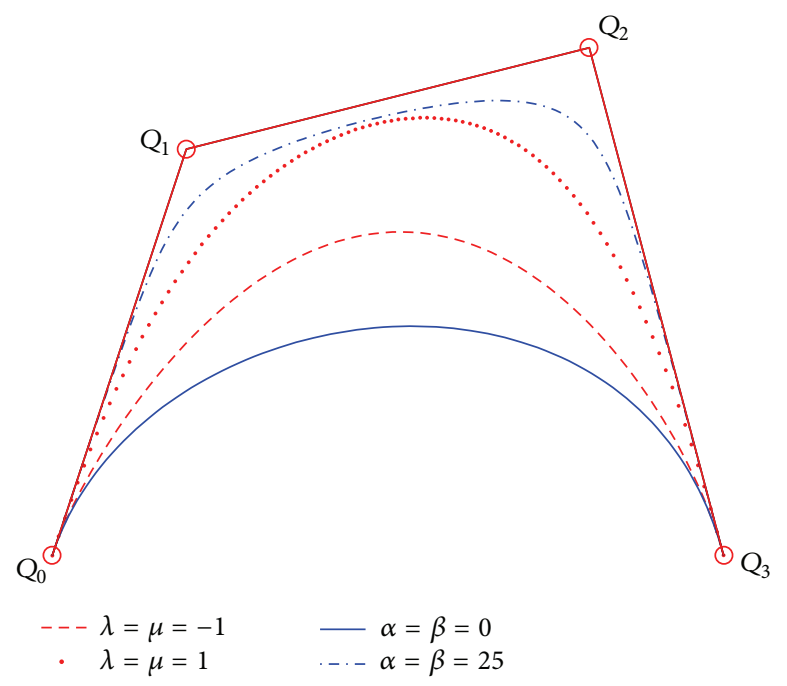

(a)

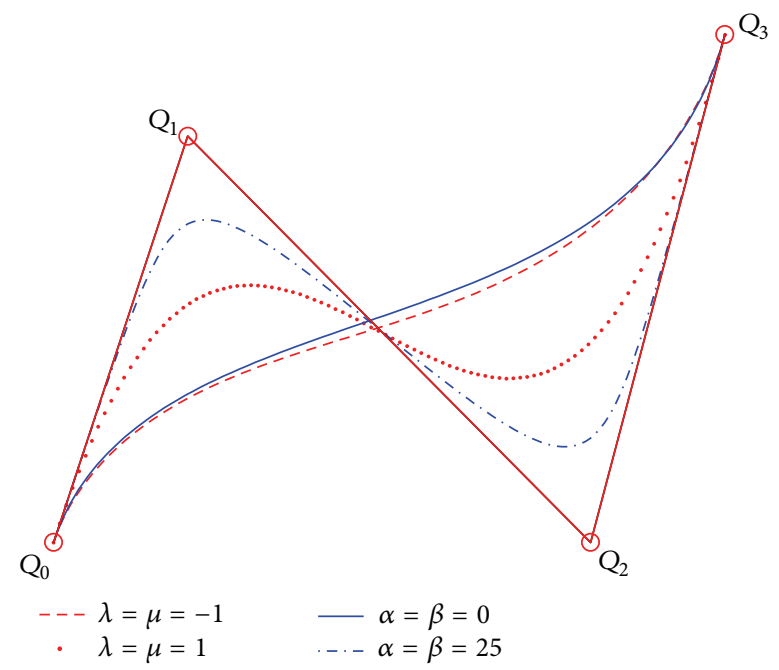

(b)

Figure 5: Comparisons between the quartic rational Said-Ball-like curves and the cubic trigonometric Bézier curves with two shape parameters $\lambda$ and $\mu$ given in [25].

\section{3. $C^{1}$ Quartic Rational Shape Preserving Interpolation Spline}

In this section, based on the quartic rational Said-Ball-like basis given in (1), we will construct a kind of $C^{1}$ quartic rational Hermite interpolation spline. Meanwhile, sufficient conditions are also derived for constructing the interpolation spline which can preserve the shape of positive or monotonic set of data.

3.1. $C^{1}$ Quartic Rational Hermite Interpolation Spline and Convergence Analysis. Let $f_{i} \in \mathbb{R}, i=1, \ldots, n$, be data given at the distinct knots $x_{i} \in \mathbb{R}, i=1, \ldots, n$, with interval spacing $h_{i}=x_{i+1}-x_{i}>0$, and let $d_{i} \in \mathbb{R}$ denote the first derivative values defined at the knots. By using the basis given in (1), we construct a quartic rational Hermite interpolation spline $H: \mathbb{R} \rightarrow \mathbb{R}$ as follows:

$$
\begin{aligned}
\left.H(x)\right|_{\left[x_{i}, x_{i+1}\right]}= & B_{0}\left(t ; \alpha_{i}\right) f_{i}+B_{1}\left(t ; \alpha_{i}\right)\left(f_{i}+\frac{h_{i}}{2+\alpha_{i}} d_{i}\right) \\
& +B_{2}\left(t ; \alpha_{i+1}\right)\left(f_{i+1}-\frac{h_{i}}{2+\alpha_{i+1}} d_{i+1}\right) \\
& +B_{3}\left(t ; \alpha_{i+1}\right) f_{i+1},
\end{aligned}
$$

where $t=\left(x-x_{i}\right) / h_{i}, \alpha_{i}, \alpha_{i+1} \in[0,+\infty), i=1,2, \ldots, n-1$.

The spline given in (11) is a $C^{1}$ Hermite interpolation spline as it satisfies the following interpolatory properties:

$$
\begin{gathered}
H\left(x_{i}\right)=f_{i}, \\
H\left(x_{i+1}\right)=f_{i+1}, \\
H^{\prime}\left(x_{i}\right)=d_{i}, \\
H^{\prime}\left(x_{i+1}\right)=d_{i+1} .
\end{gathered}
$$

And it can be easily checked that, for $\alpha_{i}=\alpha_{i+1}=0$, the quartic rational Hermite interpolation spline is exactly the classical cubic Hermite interpolation spline.

Here, all tangent information $d_{i}$ is computed from the given data $\left(x_{i}, f_{i}\right), i=1,2, \ldots, n$, as follows:

$$
\begin{aligned}
& d_{1}=\frac{f_{2}-f_{1}}{h_{1}}, \\
& d_{i}=\frac{1}{h_{i-1}+h_{i}}\left[h_{i} \frac{f_{i}-f_{i-1}}{h_{i-1}}+h_{i-1} \frac{f_{i+1}-f_{i}}{h_{i}}\right], \\
& \qquad i=2,3, \ldots, n-1, \\
& d_{n}=\frac{f_{n}-f_{n-1}}{h_{n-1}},
\end{aligned}
$$

which is used usually for generating Hermite interpolation curves.

In practical applications, the corner cutting algorithm given in (9) can be used to compute the quartic rational Hermite interpolation spline $H(x)$ effectively and stably. From (11), it is obvious that the shape parameter $\alpha_{i}$ only affects two associated curve segments. As $\alpha_{i}$ increases, the curve $H(x)$ is pulled towards the point $H\left(x_{i}\right)$ in the neighborhood of the knot position $x_{i}$. In other words, the shape parameter $\alpha_{i}$ serves as a local tension shape parameter.

Next, we will make a convergence analysis on the quartic rational Hermite interpolation spline $H(x)$. Let $f(x)$ be a given function to which $H(x)$ is compared. For $i=1,2, \ldots, n$, we denote $f_{i}=f\left(x_{i}\right), f_{i}^{\prime}=f^{\prime}\left(x_{i}\right)$ and write

$$
\begin{aligned}
\left\|f^{(4)}\right\|_{i, i+1} & =\max _{x_{i} \leq x \leq x_{i+2}}\left|f^{(4)}(x)\right|, \\
\Delta_{i} & =\frac{f_{i+1}-f_{i}}{h_{i}} .
\end{aligned}
$$


Theorem 4. Let $f \in C^{4}[a, b]$, for $x \in\left[x_{i}, x_{i+1}\right], \alpha_{i}, \alpha_{i+1} \in$ $[0,+\infty) i=1,2, \ldots, n-1$; and we have

$$
\begin{aligned}
|f(x)-H(x)| \leq & \frac{h_{i}^{4}}{384}\left\|f^{(4)}\right\|_{i, i+1} \\
& +\frac{h_{i}}{4} \max \left\{\left|f_{i}^{\prime}-d_{i}\right|,\left|f_{i+1}^{\prime}-d_{i+1}\right|\right\} \\
& +\frac{h_{i}}{8} \max \left\{\frac{\alpha_{i}^{2}\left|d_{i}\right|}{2+\alpha_{i}}, \frac{\alpha_{i+1}^{2}\left|d_{i+1}\right|}{2+\alpha_{i+1}}\right\} .
\end{aligned}
$$

Proof. For $x \in\left[x_{i}, x_{i+1}\right], i=1,2, \ldots, n-1$, let $t=\left(x-x_{i}\right) / h_{i}$ and

$$
\begin{aligned}
H_{3}(x)= & (1-t) f_{i}+t f_{i+1}-h_{i}(1-t) t \\
& \times\left[(1-t)\left(\Delta_{i}-d_{i}\right)+t\left(d_{i+1}-\Delta_{i}\right)\right] .
\end{aligned}
$$

After some computations, we have

$$
\begin{aligned}
& H(x) \\
& \begin{aligned}
=H_{3}(x)+ & {\left[\frac{\alpha_{i+1}^{2} d_{i+1}}{\left(2+\alpha_{i+1}\right)\left[1+\alpha_{i+1}(1-t)\right]}\right.} \\
& \left.-\frac{\alpha_{i}^{2} d_{i}}{\left(2+\alpha_{i}\right)\left[1+\alpha_{i} t\right]}\right](1-t)^{2} t^{2} h_{i} .
\end{aligned}
\end{aligned}
$$

With the notation $f_{i}^{\prime}=f^{\prime}\left(x_{i}\right), i=1,2, \ldots, n$, let

$$
\begin{aligned}
H_{3}^{*}(x)= & (1-t) f_{i}+t f_{i+1}-h_{i}(1-t) t \\
& \times\left[(1-t)\left(\Delta_{i}-f_{i}^{\prime}\right)+t\left(f_{i+1}^{\prime}-\Delta_{i}\right)\right] .
\end{aligned}
$$
have

Since $H_{3}^{*}(x)$ is the cubic Hermite interpolant to $f(x)$, we

$$
\left|f(x)-H_{3}^{*}(x)\right| \leq \frac{h_{i}^{4}}{384}\left\|f^{(4)}\right\|_{i, i+1} .
$$

It is easy to see that

$$
\left|H(x)-H_{3}^{*}(x)\right| \leq \frac{h_{i}}{4} \max \left\{\left|f_{i}^{\prime}-d_{i}\right|,\left|f_{i+1}^{\prime}-d_{i+1}\right|\right\},
$$

and $(1-t)^{2} t^{2} \leq 1 / 16$. From these, we can easily obtain (15).

Smooth shape preserving interpolation splines are of great significance in the area of computer graphics and scientific data visualization. In the next two subsections, we will show that a $C^{1}$ shape preserving interpolation spline for positive and/or monotonic data sets can be easily constructed by constraining the shape parameters $\alpha_{i}$ in the quartic rational Hermite spline $H(x)$ given in (11).

3.2. $C^{1}$ Positivity-Preserving Interpolation Spline. For simplicity of presentation, let us assume a positive set of data as follows:

$$
\left(x_{1}, f_{1}\right),\left(x_{2}, f_{2}\right), \ldots,\left(x_{n}, f_{n}\right),
$$

where $x_{1}<x_{2}<\cdots<x_{n}, f_{i} \in \mathbb{R}$, and $f_{i}>0, i=1,2, \ldots, n$.
Without loss of generality, we just consider the spline $H(x)$ constrained on the subinterval $\left[x_{i}, x_{i+1}\right]$. Since the quartic rational Said-Ball-like basis functions $B_{j}\left(t ; \alpha_{i}, \alpha_{i+1}\right)(j=$ $0,1,2,3)$ are nonnegative, from (11) we can see that $H(x)>0$ for any $x \in\left[x_{i}, x_{i+1}\right]$ if

$$
f_{i}+\frac{h_{i}}{2+\alpha_{i}} d_{i} \geq 0, \quad f_{i+1}-\frac{h_{i}}{2+\alpha_{i+1}} d_{i+1} \geq 0 .
$$

From (22), we can easily obtain the following sufficient conditions for constructing a $C^{1}$ positivity-preserving interpolation spline $H(x)$ :

$$
\begin{aligned}
& \alpha_{1}=\max \left\{-2-\frac{h_{1} d_{1}}{f_{1}}, 0\right\}+m_{1}, \\
& \alpha_{i}=\max \left\{-2+\frac{h_{i-1} d_{i}}{f_{i}},-2-\frac{h_{i} d_{i}}{f_{i}}, 0\right\}+m_{i}, \\
& i=2,3, \ldots, n-1, \\
& \alpha_{n}=\max \left\{-2+\frac{h_{n-1} d_{n}}{f_{n}}, 0\right\}+m_{n},
\end{aligned}
$$

where the free parameter $m_{i} \geq 0, i=1,2, \ldots, n$, can be set by users for interactively controlling the shape of the obtained spline curve.

Figure 6 shows our positivity-preserving interpolation spline (on the left) for the positive dataset given in Table 1 and the graphics of their first derivatives (on the right). It can be seen that the spline curves preserve the positive shape of the data well and they all achieve $C^{1}$ continuity. The visually pleasing positivity-preserving interpolation spline is generated by applying the sufficient conditions in (23) with different free shape parameters $m_{i}$. Figure 7 shows the comparison between our positivity-preserving interpolation spline curves ((on the left), with the choice of free parameters $m=(0,0,4,4,0,0,0,0))$ and the positivity-preserving interpolation spline curves given in [26] for the same positive dataset given in Table 2. From the results, it is obvious that our positivity-preserving interpolation spline curves and the scheme given in [26] can construct similar curves to describe the positive dataset.

3.3. $C^{1}$ Monotonicity-Preserving Interpolation Spline. Similarly as in the last subsection, a monotonic increasing set of data is given as follows:

$$
\left(x_{1}, f_{1}\right),\left(x_{2}, f_{2}\right), \ldots,\left(x_{n}, f_{n}\right),
$$

where $x_{1}<x_{2}<\cdots<x_{n}, f_{i} \in \mathbb{R}$, and $f_{1}<f_{2}<\cdots<f_{n}$. For a monotonic increasing preserving interpolation spline $H(x)$, it is necessary that the corresponding derivative values meet

$$
d_{i}>0, \quad i=1,2, \ldots, n .
$$

Obviously, the derivative values $d_{i}$ chosen by (13) can ensure this necessary condition. 


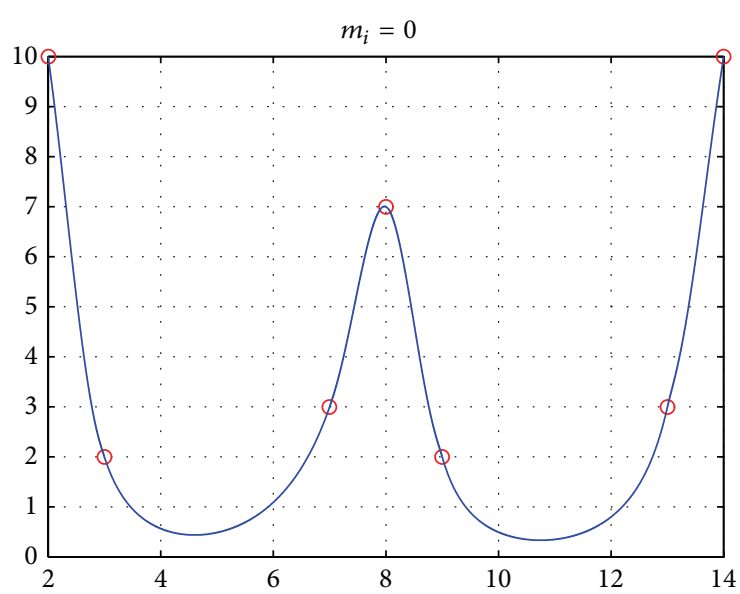

(a)

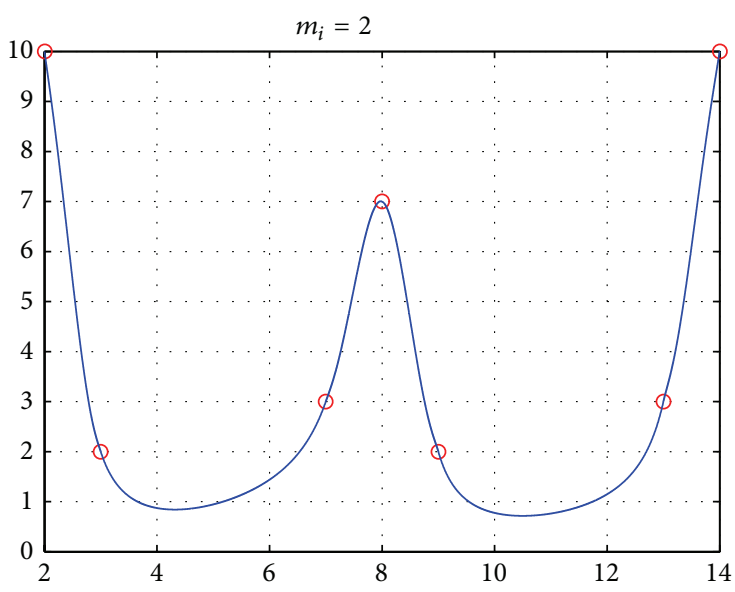

(c)

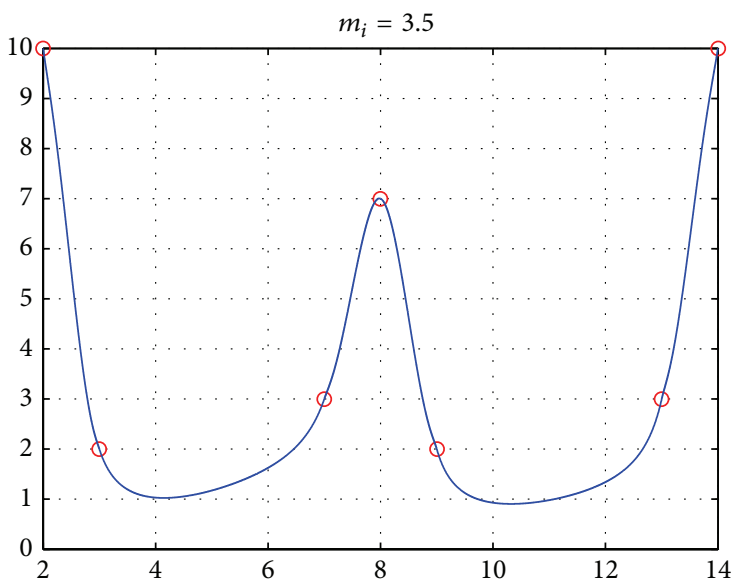

(e)

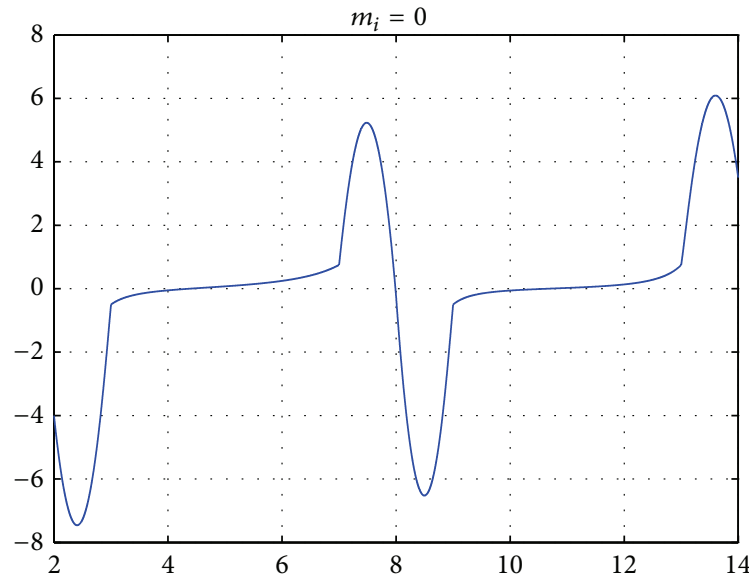

(b)

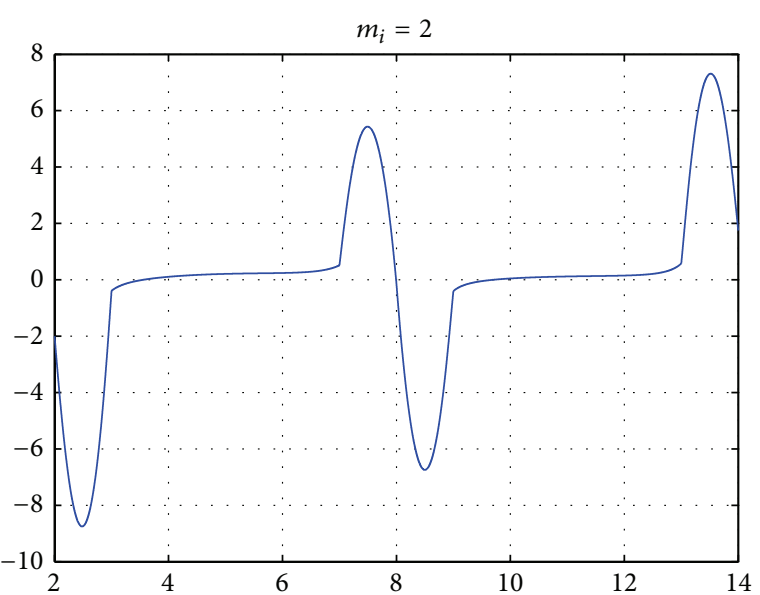

(d)

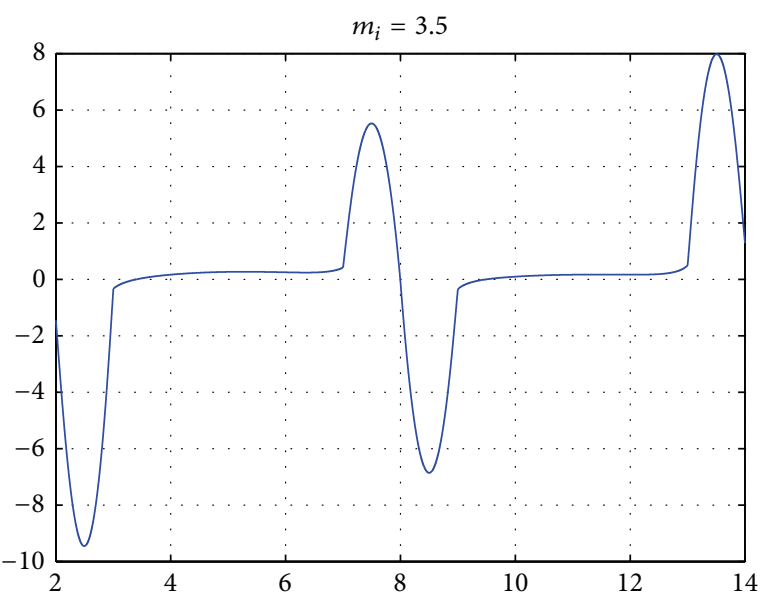

(f)

FIgURE 6: Positivity-preserving interpolation spline curves for the dataset given in Table 1 and the graphics of their first derivatives.

Without loss of generality, we just consider the function $H(x)$ constrained on the subinterval $\left[x_{i}, x_{i+1}\right]$. From (11), direct computation gives that

$$
H^{\prime}(x)=\frac{(1-t)\left(2+\alpha_{i}+\alpha_{i} t\right)}{\left(2+\alpha_{i}\right)\left(1+\alpha_{i} t\right)^{2}} d_{i}
$$

$$
\begin{aligned}
& +\frac{t\left[2+\alpha_{i+1}+\alpha_{i+1}(1-t)\right]}{\left(2+\alpha_{i+1}\right)\left[1+\alpha_{i+1}(1-t)\right]^{2}} d_{i+1} \\
& +6(1-t) t\left[\Delta_{i}-\left(\frac{d_{i}}{2+\alpha_{i}}+\frac{d_{i+1}}{2+\alpha_{i+1}}\right)\right],
\end{aligned}
$$




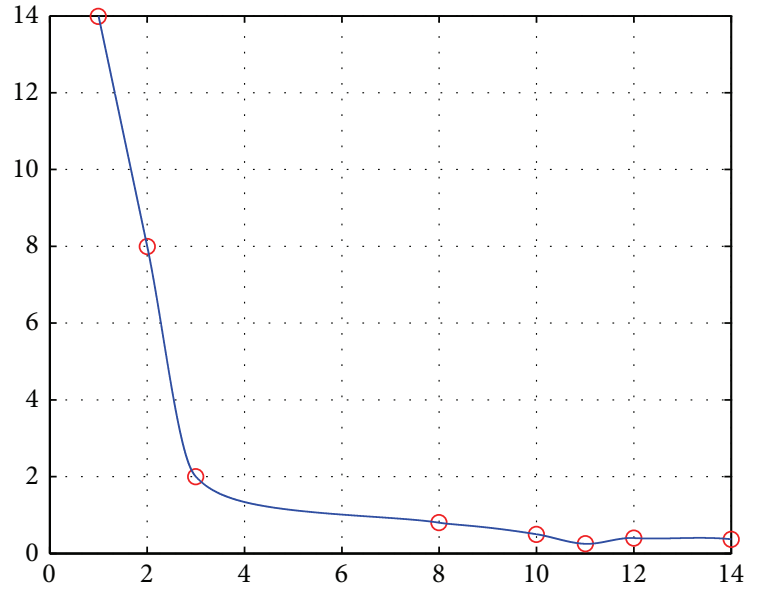

(a)

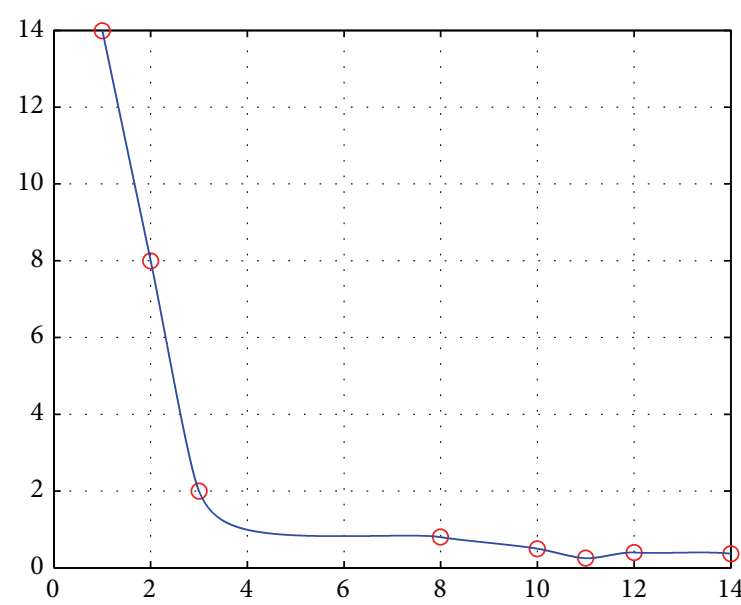

(b)

FIGURE 7: Comparison between our positivity-preserving interpolation spline curves (on the left) and the positivity-preserving interpolation spline curves given in [26].

TABLE 1: Positive dataset given in [14].

\begin{tabular}{lccccccc}
\hline$i$ & 1 & 2 & 3 & 4 & 5 & 6 & 7 \\
$x_{i}$ & 2 & 3 & 7 & 8 & 9 & 13 & 14 \\
$f_{i}$ & 10 & 2 & 3 & 7 & 2 & 3 & 10 \\
\hline
\end{tabular}

TABLE 2: Positive dataset given in [26].

\begin{tabular}{ccccccccc}
\hline$i$ & 1 & 2 & 3 & 4 & 5 & 6 & 7 & 8 \\
$x_{i}$ & 1 & 2 & 3 & 8 & 10 & 11 & 12 & 14 \\
$f_{i}$ & 14.00 & 8.00 & 2.00 & 0.80 & 0.50 & 0.25 & 0.40 & 0.37 \\
\hline
\end{tabular}

where $\Delta_{i}=\left(f_{i+1}-f\right)_{i} / h_{i}$. From $(26)$ we can see that $H^{\prime}(x)>$ 0 for any $x \in\left[x_{i}, x_{i+1}\right]$ if

$$
\Delta_{i}-\left(\frac{d_{i}}{2+\alpha_{i}}+\frac{d_{i+1}}{2+\alpha_{i+1}}\right) \geq 0,
$$

where the necessary conditions $d_{i}>0, d_{i+1}>0$ are assumed.

From (27), we can easily obtain the following sufficient conditions for constructing a $C^{1}$ monotonicity-preserving interpolation spline $H(x)$

$$
\begin{aligned}
& \alpha_{1}=\max \left\{-2+\frac{2 d_{1}}{\Delta_{1}}, 0\right\}+n_{1}, \\
& \alpha_{i}=\max \left\{-2+\frac{2 d_{i}}{\Delta_{i-1}},-2+\frac{2 d_{i}}{\Delta_{i}}, 0\right\}+n_{i}, \\
& i=2,3, \ldots, n-1, \\
& \alpha_{n}=\max \left\{-2+\frac{2 d_{n}}{\Delta_{n-1}}, 0\right\}+n_{n},
\end{aligned}
$$

where the free parameter $n_{i} \geq 0, i=1,2, \ldots, n$, can be specified by users to achieve a monotonicity-preserving interpolation spline.

Similarly, we can deal with a monotonic decreasing data. It should be noted that, if $f_{i+1}=f_{i}$, it is necessary to set $f_{i}=f_{i+1}=0$. Thus for any $\alpha_{i}, \alpha_{i+1} \in[0,+\infty), H(x)=$ $f_{i}=f_{i+1}$, is a constant on $\left[x_{i}, x_{i+1}\right]$. For the case where the data is monotonic but not strictly monotonic (i.e., when some $f_{i+1}=f_{i}$ ) it would be necessary to divide the data into several strictly monotonic parts. And we should set $d_{i}=d_{i+1}=0$, whenever $f_{i+1}=f_{i}$.

Figure 8 shows our monotonicity-preserving interpolation spline (on the left) for the dataset in Table 3 and the graphics of their first derivatives (on the right). Obviously, all of the interpolation spline curves have the properties of monotonicity-preserving and they are all $C^{1}$ continuous. The visually pleasing monotonicity-preserving interpolation spline is generated by applying the sufficient conditions in (28) with different free shape parameters $n_{i}$. Figure 9 shows the comparison between our monotonicity-preserving interpolation spline curves (on the left, with the choice of the free parameters $n=(0,0,0,0))$ and the monotonicitypreserving interpolation spline curves given in [26] for the monotone dataset given in Table 4 . From the results, it can be seen that our monotonicity-preserving interpolation spline curves describe the monotone dataset as well as the scheme given in [26].

\section{Quartic Rational Said-Ball-Like Basis over a Triangular Domain}

Based on the proposed quartic rational Said-Ball-like basis with two shape parameters given in (1), by using the method of tensor product, we can easily construct a kind of biquartic rational Said-Ball-like patch over a rectangle domain with four shape parameters. However, the patch over the triangular 


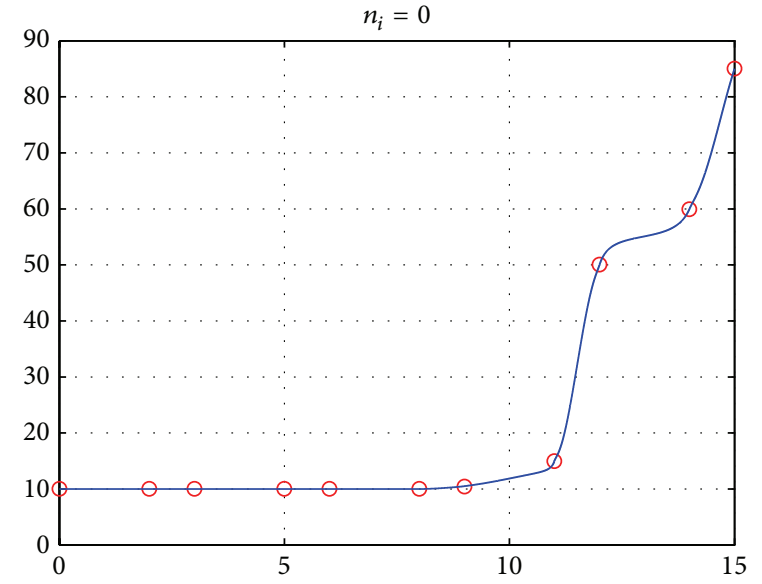

(a)

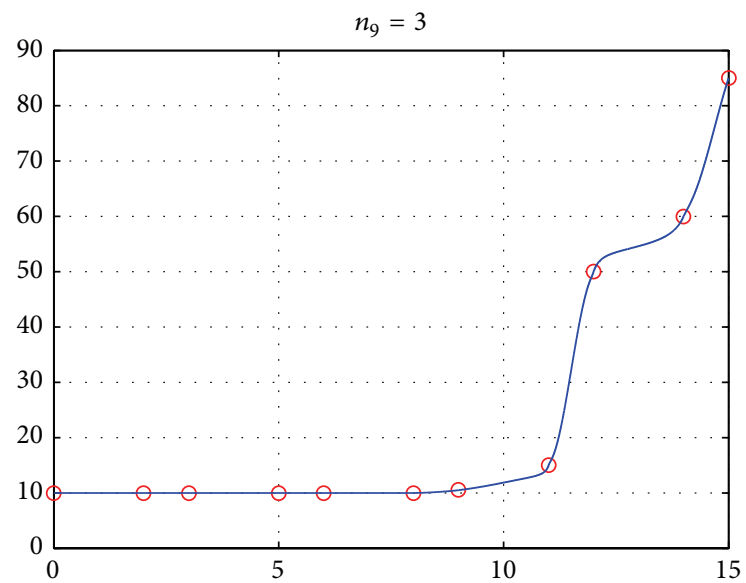

(c)

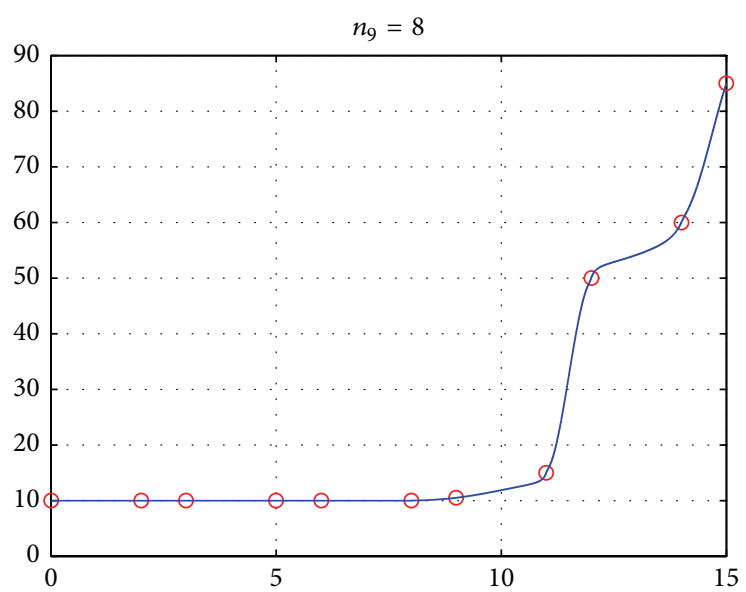

(e)

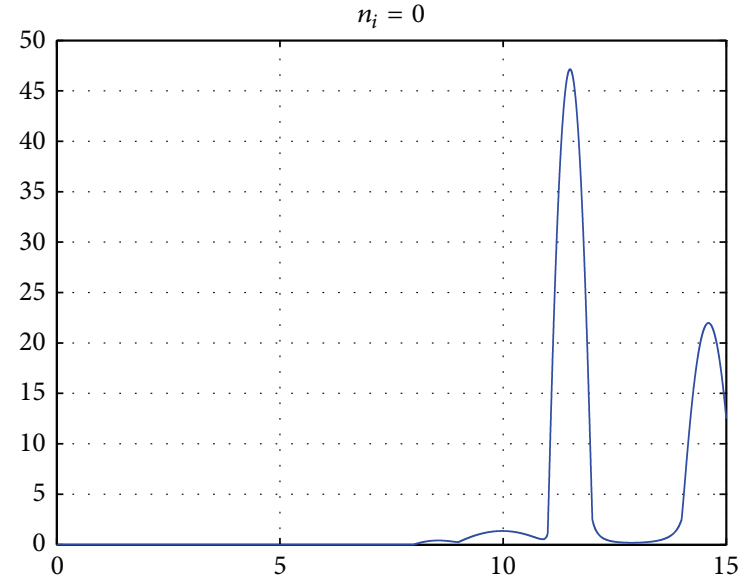

(b)

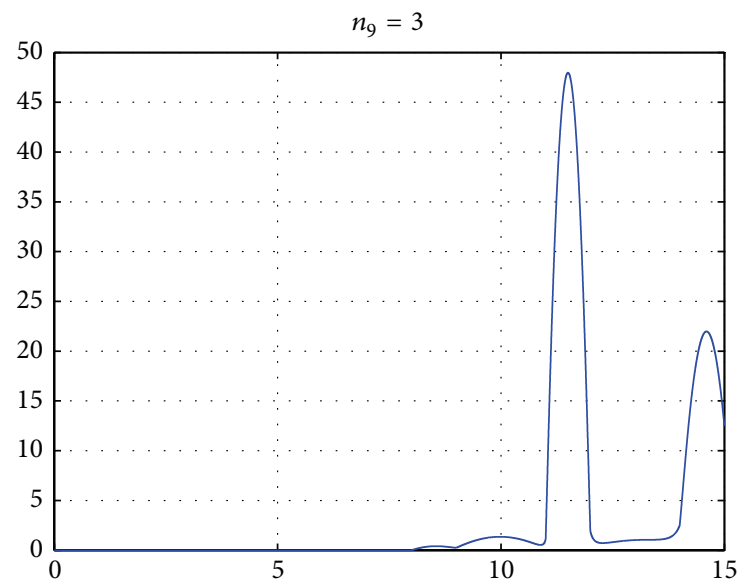

(d)

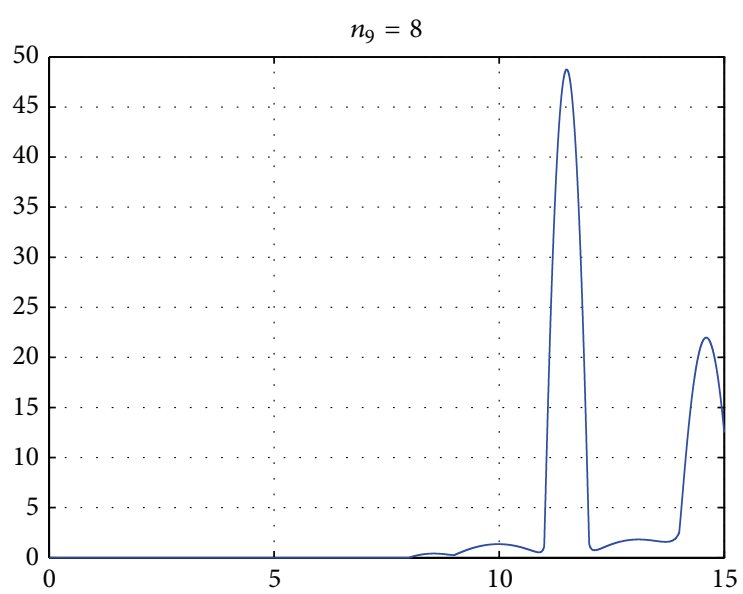

(f)

FIGURE 8: Monotonicity-preserving interpolation spline curves for the Akima's data given in Table 3 and the graphics of their first derivatives.

domain is not a tensor product patch exactly. These imply that we cannot extend the basis (1) to a triangular domain by using the method of tensor product. In this section, we will construct a new class of quartic rational Said-Ball-like basis over a triangular domain with three shape parameters, which is an extension over a triangular domain of the basis (1).
4.1. Construction and Properties of the Basis. Here, we give the definition of the new quartic rational Said-Ball-like basis functions over a triangular domain as follows.

Definition 5. Let $\alpha, \beta, \gamma \in[0,+\infty)$, and $D:=\{(t, s, w) \mid t+$ $s+w=1, t \geq 0, s \geq 0, w \geq 0\}$; the following ten functions are 


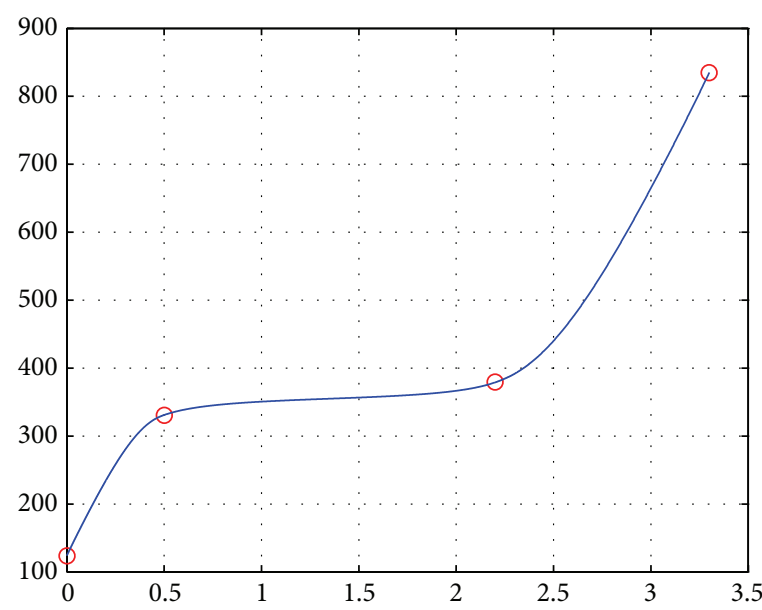

(a)

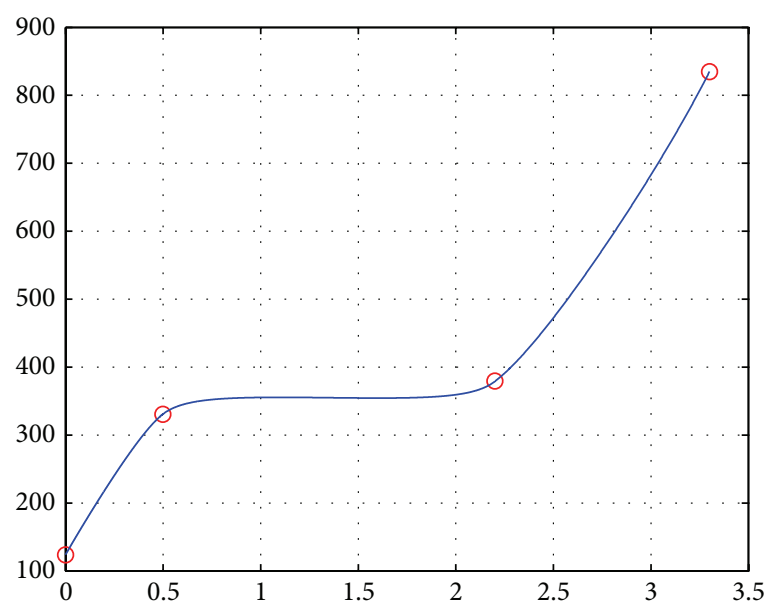

(b)

FIGURE 9: Comparison between our monotonicity-preserving interpolation spline curves (on the left) and the monotonicity-preserving interpolation spline curves given in [26].

TABLE 3: Akima's dataset given in [27].

\begin{tabular}{cccccccccccc}
\hline$i$ & 1 & 2 & 3 & 4 & 5 & 6 & 7 & 8 & 9 & 10 & 11 \\
$x_{i}$ & 0 & 2 & 3 & 5 & 6 & 8 & 9 & 11 & 12 & 14 & 15 \\
$f_{i}$ & 10 & 10 & 10 & 10 & 10 & 10 & 10.5 & 15 & 50 & 60 & 85 \\
\hline
\end{tabular}

TABLE 4: The monotone dataset given in [26].

\begin{tabular}{ccccc}
\hline$i$ & 1 & 2 & 3 & 4 \\
$x_{i}$ & 0.0 & 0.5 & 2.2 & 3.3 \\
$f_{i}$ & 124 & 331 & 379 & 835 \\
\hline
\end{tabular}

defined as new quartic rational Said-Ball-like basis functions, with three shape parameters $\alpha, \beta$, and $\gamma$, over the triangular domain $D$ :

$$
\begin{aligned}
& B_{3,0,0}^{3}(t, s, w)=\frac{t^{2}}{1+\alpha(1-t)}, \\
& B_{0,3,0}^{3}(t, s, w)=\frac{s^{2}}{1+\beta(1-s)}, \\
& B_{0,0,3}^{3}(t, s, w)=\frac{w^{2}}{1+\gamma(1-w)}, \\
& B_{2,1,0}^{3}(t, s, w)=\frac{t^{2} s[2+\alpha+2 \alpha(1-t)]}{1+\alpha(1-t)}, \\
& B_{2,0,1}^{3}(t, s, w)=\frac{t^{2} w[2+\alpha+2 \alpha(1-t)]}{1+\alpha(1-t)}, \\
& B_{1,2,0}^{3}(t, s, w)=\frac{t s^{2}[2+\beta+2 \beta(1-s)]}{1+\beta(1-s)}, \\
& B_{0,2,1}^{3}(t, s, w)=\frac{s^{2} w[2+\beta+2 \beta(1-s)]}{1+\beta(1-s)},
\end{aligned}
$$

$$
\begin{aligned}
& B_{1,0,2}^{3}(t, s, w)=\frac{t w^{2}[2+\gamma+2 \gamma(1-w)]}{1+\gamma(1-w)}, \\
& B_{0,1,2}^{3}(t, s, w)=\frac{s w^{2}[2+\gamma+2 \gamma(1-w)]}{1+\gamma(1-w)}, \\
& B_{1,1,1}^{3}(t, s, w)=1-\sum_{\substack{i+j+k=3, i \cdot j \cdot k \neq 1}} B_{i, j, k}^{3}(t, s, w) .
\end{aligned}
$$

Remark 6. In the following discussion, we will denote the new quartic rational Said-Ball-like basis functions as $B_{i, j, k}^{3}(t, s, w ; \alpha, \beta, \gamma), i, j, k \in \mathbb{N}, i+j+k=3$. It is easy to check that for $\alpha=\beta=\gamma=0$, the basis functions given in (29) are precisely the cubic Said-Ball basis functions over a triangular domain; see [8].

From the expressions of the new quartic rational SaidBall-like basis functions over a triangular domain given in (29), we can obtain the following important properties of the new basis.

Theorem 7. The basis functions over a triangular domain given in (29) have the following properties.

(a) Partition of unity: $\sum_{i+j+k=3} B_{i, j, k}^{3}(t, s, w)=1$.

(b) Nonnegativity: $B_{i, j, k}^{3}(t, s, w) \geq 0, i, j, k \in \mathbb{N}, i+j+k=$ 3.

(c) Property at the corner points: for $i, j, k \in \mathbb{N}, i+j+k=3$, we have

$$
\begin{aligned}
& B_{i, j, k}^{3}(1,0,0)= \begin{cases}1, & i=3, \\
0, & i \neq 3,\end{cases} \\
& B_{i, j, k}^{3}(0,1,0)= \begin{cases}1, & j=3, \\
0, & j \neq 3,\end{cases} \\
& B_{i, j, k}^{3}(0,0,1)= \begin{cases}1, & k=3, \\
0, & k \neq 3 .\end{cases}
\end{aligned}
$$


(d) Derivative at the corner points: for $t, s, 1-t-s \geq 0$, $i, j, k \in \mathbb{N}$, and $i+j+k=3$, we have

$$
\begin{aligned}
& \left.\frac{\partial B_{i, j, k}^{3}(t, s, 1-t-s)}{\partial t}\right|_{(1,0,0)}= \begin{cases}2+\alpha, & (i, j, k)=(3,0,0), \\
-2-\alpha, & (i, j, k)=(2,0,1), \\
0, & \text { other, }\end{cases} \\
& \left.\frac{\partial B_{i, j, k}^{3}(t, s, 1-t-s)}{\partial s}\right|_{(1,0,0)}= \begin{cases}2+\alpha, & (i, j, k)=(2,1,0), \\
-2-\alpha, & (i, j, k)=(2,0,1), \\
0, & \text { other, }\end{cases}
\end{aligned}
$$$$
\left.\frac{\partial B_{i, j, k}^{3}(t, s, 1-t-s)}{\partial t}\right|_{(0,1,0)}= \begin{cases}2+\beta, & (i, j, k)=(1,2,0), \\ -2-\beta, & (i, j, k)=(0,2,1), \\ 0, & \text { other, }\end{cases}
$$$$
\left.\frac{\partial B_{i, j, k}^{3}(t, s, 1-t-s)}{\partial s}\right|_{(0,1,0)}= \begin{cases}2+\beta, & (i, j, k)=(0,3,0), \\ -2-\beta, & (i, j, k)=(0,2,1), \\ 0, & \text { other, }\end{cases}
$$$$
\left.\frac{\partial B_{i, j, k}^{3}(t, s, 1-t-s)}{\partial t}\right|_{(0,0,1)}= \begin{cases}2+\gamma, & (i, j, k)=(1,0,2), \\ -2-\gamma, & (i, j, k)=(0,0,3), \\ 0, & \text { other, }\end{cases}
$$$$
\left.\frac{\partial B_{i, j, k}^{3}(t, s, 1-t-s)}{\partial s}\right|_{(0,0,1)}= \begin{cases}2+\gamma, & (i, j, k)=(0,1,2), \\ -2-\gamma, & (i, j, k)=(0,0,3), \\ 0, & \text { other. }\end{cases}
$$

(e) Boundary property: when one of the three variables $w$ is taken as zero, the ten quartic rational basis functions $B_{i, j, k}^{3}(t, s, w)$ degenerate to the corresponding quartic rational Said-Ball-like basis functions $B_{i}(t ; \beta, \alpha)$ (notice $s=1-t$ ) given in (1).

(f) Linear independence: $\left\{B_{i, j, k}^{3}(t, s, w), j+j+k=3\right\}$ are linearly independent.

Proof. We will prove (b) and (f). Simple and direct computation can give out the remaining properties.

(b) For any $\alpha, \beta, \gamma \in[0,+\infty), i, j, k \in \mathbb{N}, i+j+k=3$, and $i \cdot j \cdot k \neq 1, B_{i, j, k}^{3}(t, s, w) \geq 0$ is obvious. Furthermore, for $B_{1,1,1}^{3}(t, s, w)$, by directly computing, we have

$$
\begin{aligned}
B_{1,1,1}^{3}(t, s, w) & =1-\sum_{\substack{i+j+k=3, i \cdot j \cdot k \neq 1}} B_{i, j, k}^{3}(t, s, w) \\
& =1-\left[3\left(t^{2}+s^{2}+w^{2}\right)-2\left(t^{3}+s^{3}+w^{3}\right)\right] \\
& =6 t s w \geq 0 .
\end{aligned}
$$

(f) For $\alpha, \beta, \gamma \in[0,+\infty), \xi_{i, j, k} \in \mathbb{R}(i+j+k=3)$, we consider a linear combination

$$
\sum_{i+j+k=3} \xi_{i, j, k} B_{i, j, k}^{3}(t, s, w)=0 .
$$

Let $w=0$; we have

$$
\sum_{i=0}^{3} \xi_{i, 3-i, 0} B_{i}(t ; \beta, \alpha)=0 .
$$
we have

Differentiating with respect to the variable $t$ on both sides,

$$
\sum_{i=0}^{3} \xi_{i, 3-i, 0} B_{i}^{\prime}(t ; \beta, \alpha)=0 .
$$

For $t=0$, from (34) and (35), we get

$$
\begin{gathered}
\xi_{0,3,0}=0, \\
(2+\beta)\left(\xi_{1,2,0}-\xi_{0,3,0}\right)=0 .
\end{gathered}
$$

Thus, we have $\xi_{0,3,0}=\xi_{1,2,0}=0$. And for $t=1$, from (34) and (35), we can also obtain $\xi_{3,0,0}=\xi_{2,1,0}=0$. Similarly, $\xi_{i, 0,3-i}=\xi_{0, i, 3-i}=0, i=0,1,2,3$. And finally, $\xi_{1,1,1}=0$.

These imply the theorem.

Figure 10 shows some plots of quartic rational Said-Balllike basis functions over a triangular domain. The three shape parameters take values $\alpha=0, \beta=1, \gamma=2$.

\subsection{Definition and Properties of the Quartic Rational Triangu- lar Said-Ball-Like Patch}

Definition 8. Let $\alpha, \beta, \gamma \in[0,+\infty)$, given control points $P_{i j k} \in$ $\mathbb{R}^{3}(i, j, k \in \mathbb{N}, i+j+k=3)$ and a triangular domain $D=$ $\{(t, s, w) \mid t+s+w=1, t \geq 0, s \geq 0, w \geq 0\}$. We call

$$
R(t, s, w)=\sum_{i+j+k=3} B_{i, j, k}^{3}(t, s, w) P_{i, j, k}, \quad(t, s, w) \in D
$$

the quartic rational triangular Said-Ball-like patch with three shape parameters $\alpha, \beta, \gamma$.

From the properties of the quartic rational Said-Ball-like basis functions over a triangular domain, some properties of the quartic rational triangular Said-Ball-liek patch (37) can be obtained easily as follows.

(a) Affine invariance and convex hull properties: since the basis functions (29) have the properties of partition of unity and nonnegativity, we can see that the corresponding patch (37) has affine invariance and convex hull property.

(b) Geometric properties at the corner points: direct computation gives that

$$
\begin{gathered}
R(1,0,0)=P_{3,0,0}, \quad R(0,1,0)=P_{0,3,0}, \\
R(0,0,1)=P_{0,0,3} .
\end{gathered}
$$

These indicate that the patch (37) interpolates at the corner points. 


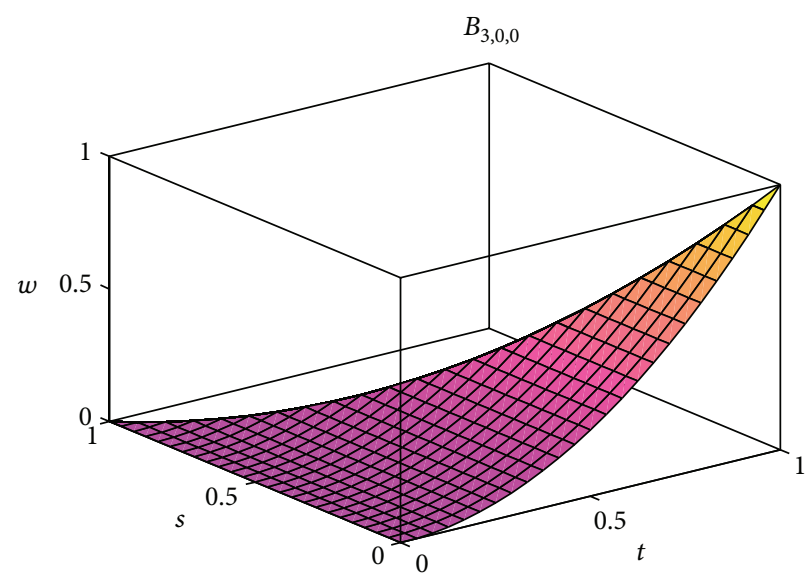

(a)

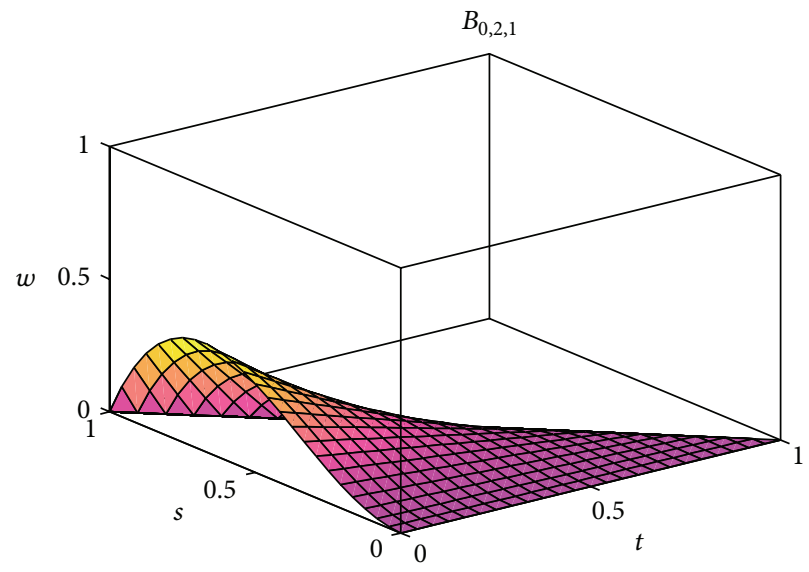

(c)

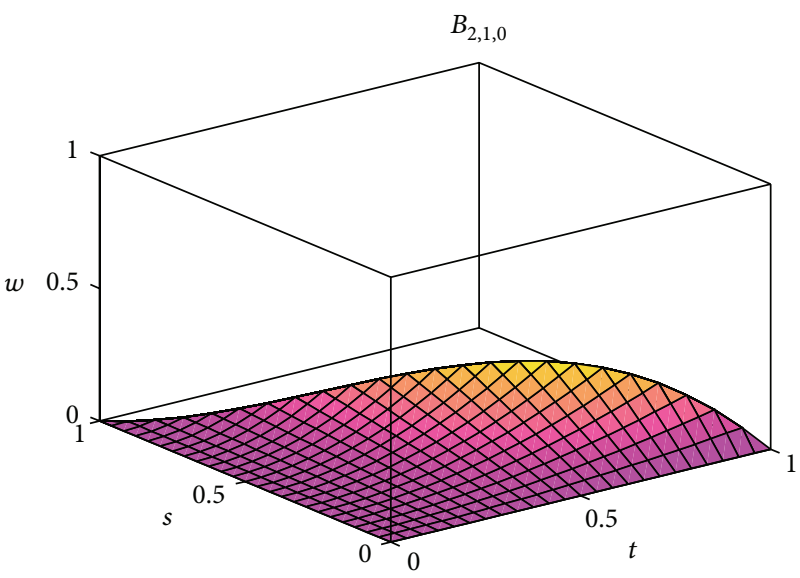

(b)

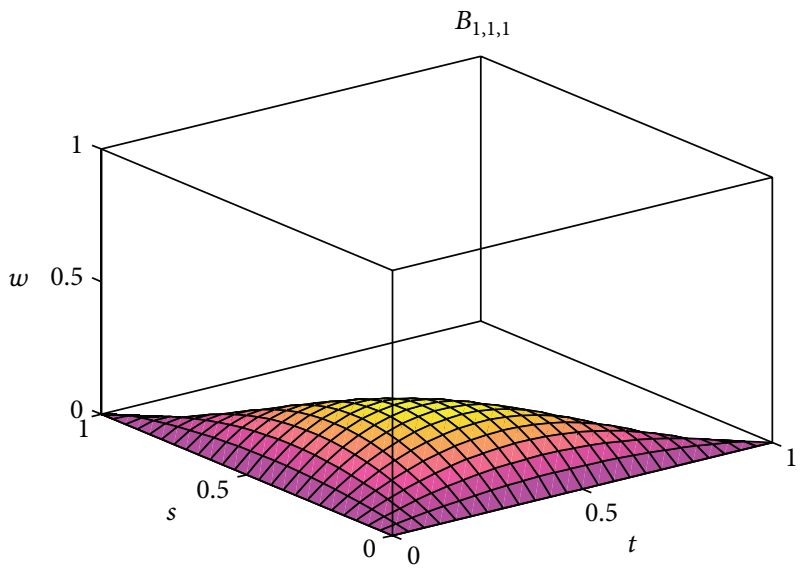

(d)

FIGURE 10: Some plots of quartic rational Said-Ball-like basis functions over a triangular domain.

(c) Tangent planes at the corner points: let $w=1-s-t$; we have

$$
\begin{aligned}
& \left.\frac{\partial R(t, s, w)}{\partial t}\right|_{(1,0,0)}=(2+\alpha)\left(P_{3,0,0}-P_{2,0,1}\right), \\
& \left.\frac{\partial R(t, s, w)}{\partial s}\right|_{(1,0,0)}=(2+\alpha)\left(P_{2,1,0}-P_{2,0,1}\right), \\
& \left.\frac{\partial R(t, s, w)}{\partial t}\right|_{(0,1,0)}=(2+\beta)\left(P_{1,2,0}-P_{0,2,1}\right), \\
& \left.\frac{\partial R(t, s, w)}{\partial s}\right|_{(0,1,0)}=(2+\beta)\left(P_{0,3,0}-P_{0,2,1}\right), \\
& \left.\frac{\partial R(t, s, w)}{\partial t}\right|_{(0,0,1)}=(2+\gamma)\left(P_{1,0,2}-P_{0,0,3}\right), \\
& \left.\frac{\partial R(t, s, w)}{\partial s}\right|_{(0,0,1)}=(2+\gamma)\left(P_{0,1,2}-P_{0,0,3}\right) .
\end{aligned}
$$

These indicate that the tangent planes at the three corner points $(1,0,0),(0,1,0)$, and $(0,0,1)$ are the three planes spanned by the control points $P_{3,0,0}$, $P_{2,1,0}$, and $P_{2,0,1} ; P_{0,3,0}, P_{1,2,0}$, and $P_{0,2,1}$; and $P_{0,0,3}$, $P_{1,0,2}$, and $P_{0,1,2}$, respectively.

(d) Boundary property: for $w=0, R(t, s, w)$ is a quartic rational Said-Ball-like curve with two shape parameters $\alpha$ and $\beta$. And similarly, $R(0, s, w)$ and $R(t, 0, w)$ both are quartic rational Said-Ball-like curves with shape parameters $\beta, \gamma$ and $\alpha, \gamma$, respectively.

(e) Shape adjustable property: without changing the control net, we can adjust the shape of the quartic rational triangular Said-Ball-like patch conveniently by using the three shape parameters $\alpha, \beta$, and $\gamma$. As the three shape parameters increase at the same time, the patch will be made close to the control net. Thus the three shape parameters $\alpha, \beta, \gamma$ serve as tension parameters. Furthermore, from the boundary property of the patch, we can see that the three shape parameters $\alpha, \beta$, and $\gamma$ have nothing to do with the boundary 
curves $R(0, s, w), R(t, 0, w)$, and $R(t, s, 0)$, respectively. These imply that altering the value of a single shape parameter, one corresponding boundary curve of the generated patch will not change.

Figure 11 shows the quartic rational triangular patches and the effect on the patches by altering the values of the shape parameters without changing the control points.

4.3. De Casteljau-Type Algorithm. The classical de Casteljau algorithm is a stable and efficient process for computing the triangular Bernstein-Bézier patch. Here, we will develop a de Casteljau-type algorithm for computing the proposed quartic rational triangular Said-Ball-like patch. By setting

$$
\begin{aligned}
P_{2,0,0}^{1}:= & \frac{1}{1+\alpha(1-t)} P_{3,0,0}+\frac{\alpha s}{1+\alpha(1-t)} P_{2,1,0} \\
& +\frac{\alpha w}{1+\alpha(1-t)} P_{2,0,1}, \\
& P_{1,1,0}^{1}:=t P_{2,1,0}+s P_{1,2,0}+w P_{1,1,1} \\
& P_{1,0,1}^{1}:=t P_{2,0,1}+s P_{1,1,1}+w P_{1,0,2}, \\
& P_{1,1,0}^{1}:=t P_{2,1,0}+s P_{1,2,0}+w P_{1,1,1}, \\
P_{0,2,0}^{1}:= & \frac{\beta t}{1+\beta(1-s)} P_{1,2,0}+\frac{1}{1+\beta(1-s)} P_{0,3,0} \\
& +\frac{\beta w}{1+\beta(1-s)} P_{0,2,1}, \\
& P_{0,1,1}^{1}:=t P_{1,1,1}+s P_{0,2,1}+w P_{0,1,2}, \\
& P_{1,0,1}^{1}:=t P_{2,0,1}+s P_{1,1,1}+w P_{1,0,2}, \\
& P_{0,1,1}^{1}:=t P_{1,1,1}+s P_{0,2,1}+w P_{0,1,2}, \\
P_{0,0,2}^{1}:= & \frac{\gamma t}{1+\gamma(1-w)} P_{1,0,2}+\frac{\gamma s}{1+\gamma(1-w)} P_{0,1,2} \\
& +\frac{1}{1+\gamma(1-w)} P_{0,0,3},
\end{aligned}
$$

we have

$$
\begin{aligned}
R(t, s, w)= & t\left(t P_{2,0,0}^{1}+s P_{1,1,0}^{1}+w P_{1,0,1}^{1}\right) \\
& +s\left(t P_{1,1,0}^{1}+s P_{0,2,0}^{1}+w P_{0,1,1}^{1}\right) \\
& +w\left(t P_{1,0,1}^{1}+s P_{0,1,1}^{1}+w P_{0,0,2}^{1}\right)
\end{aligned}
$$

Furthermore, there are three points at the second level:

$$
\begin{aligned}
& P_{1,0,0}^{2}:=t P_{2,0,0}^{1}+s P_{1,1,0}^{1}+w P_{1,0,1}^{1}, \\
& P_{0,1,0}^{2}:=t P_{1,1,0}^{1}+s P_{0,2,0}^{1}+w P_{0,1,1}^{1}, \\
& P_{0,0,1}^{2}:=t P_{1,0,1}^{1}+s P_{0,1,1}^{1}+w P_{0,0,2}^{1},
\end{aligned}
$$

which lead to

$$
R(t, s, w)=t P_{1,0,0}^{2}+s P_{0,1,0}^{2}+w P_{0,0,1}^{2}:=P_{0,0,0}^{3} .
$$

From (41) and (43), we can immediately obtain the following de Casteljau-type algorithm:

$$
\begin{aligned}
P_{i, j, k}^{m}= & f_{i+1, j, k}^{m-1}(t, s, w) P_{i+1, j, k}^{m-1}+g_{i, j+1, k}^{m-1}(t, s, w) \\
& \times P_{i, j+1, k}^{m-1}+h_{i, j, k+1}^{m-1}(t, s, w) P_{i, j, k+1}^{m-1},
\end{aligned}
$$

where $m=1,2,3, i+j+k=3-m, i, j, k \geq 0, P_{i, j, k}^{0}=P_{i, j, k}$ and

$$
\begin{aligned}
& f_{i+1, j, k}^{m-1}(t, s, w)= \begin{cases}\frac{1}{1+\alpha(1-t)}, & (m, i, j, k)=(1,2,0,0), \\
\frac{\beta t}{1+\beta(1-s)}, & (m, i, j, k)=(1,0,2,0), \\
\frac{\gamma t}{1+\gamma(1-w)}, & (m, i, j, k)=(1,0,0,2), \\
t, & \text { other, }\end{cases} \\
& g_{i, j+1, k}^{m-1}(t, s, w)= \begin{cases}\frac{\alpha s}{1+\alpha(1-t)}, & (m, i, j, k)=(1,2,0,0), \\
\frac{1}{1+\beta(1-s)}, & (m, i, j, k)=(1,0,2,0), \\
\frac{\gamma s}{1+\gamma(1-w)}, & (m, i, j, k)=(1,0,0,2), \\
s, & \text { other, }\end{cases} \\
& h_{i, j, k+1}^{m-1}(t, s, w)= \begin{cases}\frac{\alpha w}{1+\alpha(1-t)}, & (m, i, j, k)=(1,2,0,0), \\
\frac{\beta w}{1+\beta(1-s)}, & (m, i, j, k)=(1,0,2,0), \\
\frac{1}{1+\gamma(1-w)}, & (m, i, j, k)=(1,0,0,2), \\
w, & \text { other. }\end{cases}
\end{aligned}
$$

4.4. Joining Two Patches. In practical surface construction, we often need to join several patches together in order to generate shapes that are too complex to handle with a single patch. During the joining of quartic rational triangular SaidBall-like patches, we need to control the smoothness of the connecting surface. For $(t, s, w) \in D$, let two quartic rational triangular Said-Ball-like patches be defined as

$$
\begin{aligned}
& R_{1}(t, s, w)=\sum_{i+j+k=3} B_{i, j, k}^{3}\left(t, s, w ; \alpha_{1}, \beta, \gamma\right) P_{i, j, k}, \\
& R_{2}(t, s, w)=\sum_{i+j+k=3} B_{i, j, k}^{3}\left(t, s, w ; \alpha_{2}, \beta, \gamma\right) Q_{i, j, k} .
\end{aligned}
$$

It is apparent that if the control points satisfy

$$
P_{0, j, k}=Q_{0, j, k}, \quad j, k \in \mathbb{N}, j+k=3,
$$

the two patches will join along a common boundary curve: $R_{1}(0, s, w)=R_{2}(0, s, w), s+w=1$. Thus the two patches clearly form a surface with positional continuity or a surface with $C^{0}$ continuity. 


$$
\alpha=0, \beta=0, \gamma=0
$$

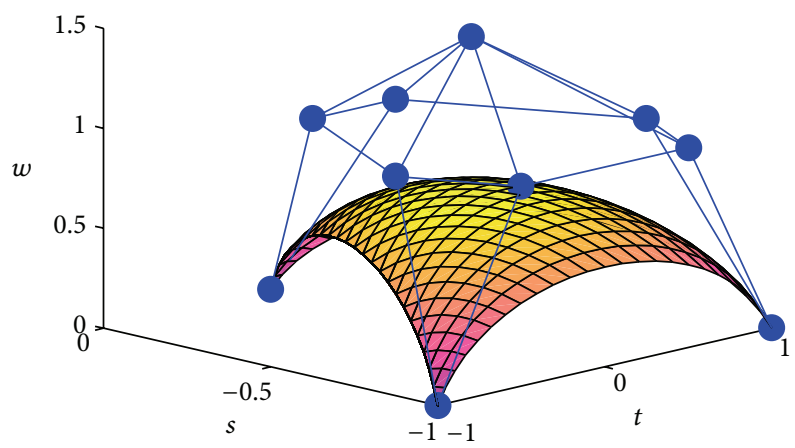

(a)

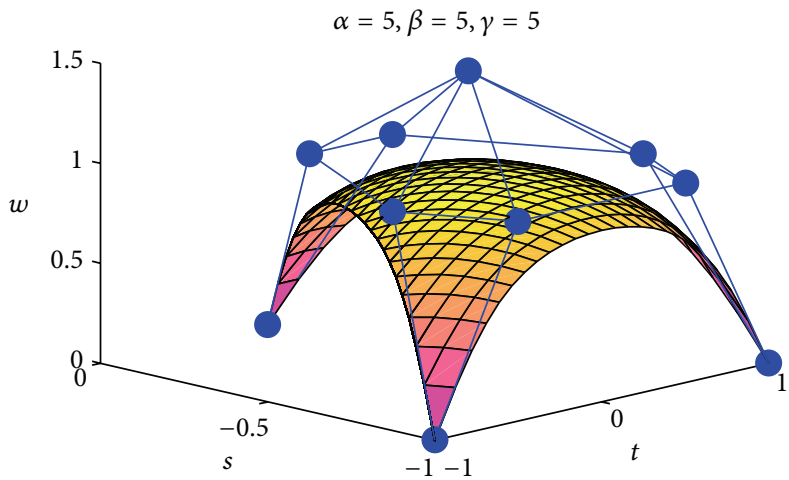

(c)

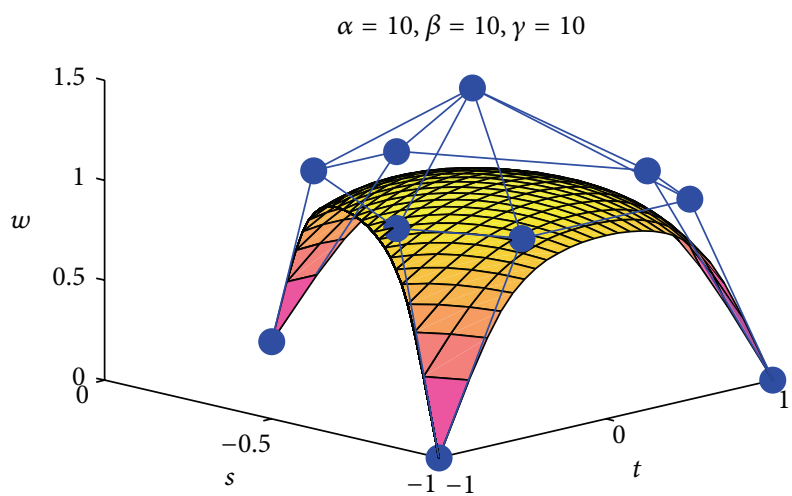

(e)

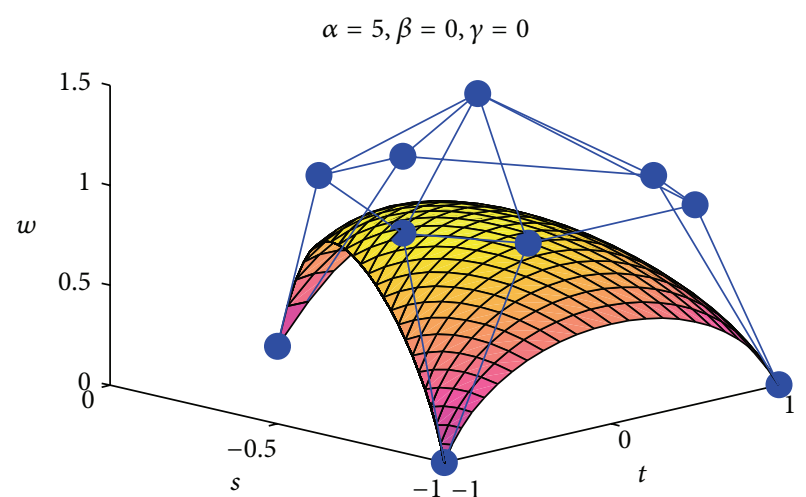

(b)

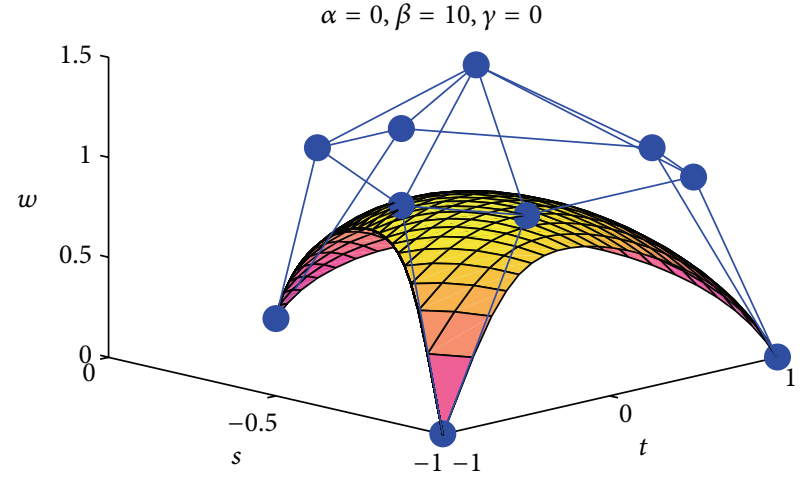

(d)

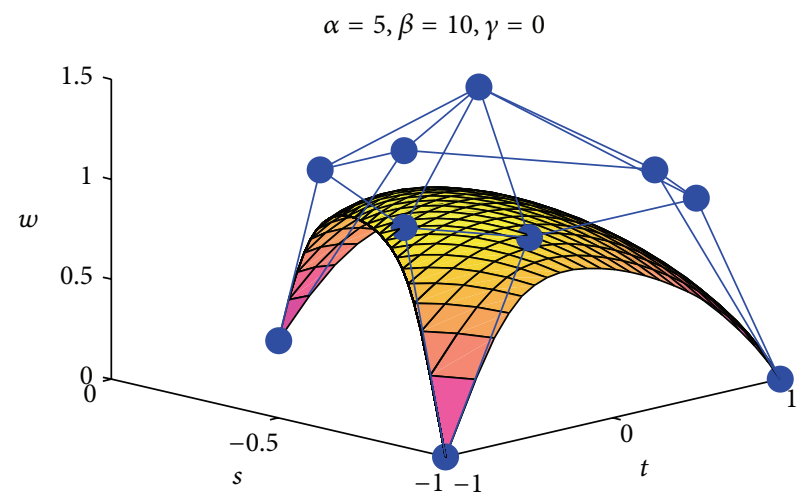

(f)

FIGURE 11: Quartic rational triangular Said-Ball-like patches with different shape parameters.

For the common boundary curve $R_{1}(0, s, 1-s)$, differentiating with respect to $s$, we have

$$
\begin{aligned}
& \frac{d R_{1}(0, s, 1-s)}{d s} \\
& =\frac{s[2+\beta+\beta(1-s)]}{[1+\beta(1-s)]^{2}}\left(P_{0,3,0}-P_{0,2,1}\right) \\
& +6 s(1-s)\left(P_{0,2,1}-P_{0,1,2}\right) \\
& +\frac{(1-s)[2+\gamma+\gamma s]}{[1+\gamma s]^{2}}\left(P_{0,1,2}-P_{0,0,3}\right) .
\end{aligned}
$$

For $R_{1}(t, s, 1-t-s)$ and $R_{2}(t, s, 1-t-s)$, we do differentiate with respect to $t$

$$
\begin{aligned}
\left.\frac{\partial R_{1}(t, s, 1-t-s)}{\partial t}\right|_{t=0} & \\
= & \frac{s[2+\beta+\beta(1-s)]}{[1+\beta(1-s)]^{2}}\left(P_{1,2,0}-P_{0,2,1}\right) \\
& +6 s(1-s)\left(P_{1,1,1}-P_{0,1,2}\right) \\
& +\frac{(1-s)[2+\gamma+\gamma s]}{[1+\gamma s]^{2}}\left(P_{1,0,2}-P_{0,0,3}\right),
\end{aligned}
$$




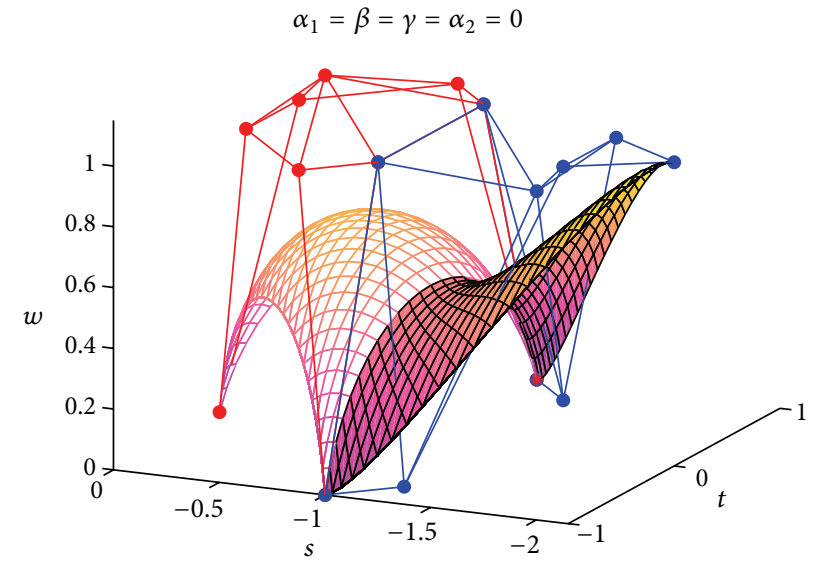

(a)

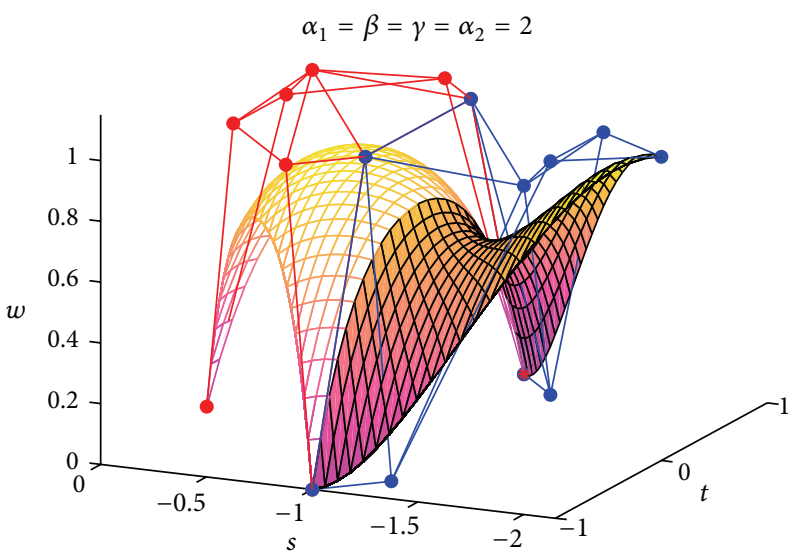

(c)

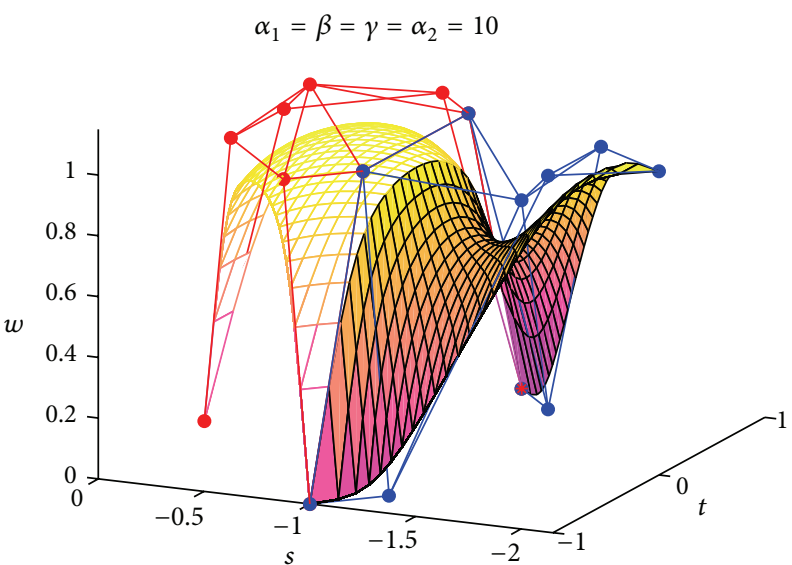

(e)

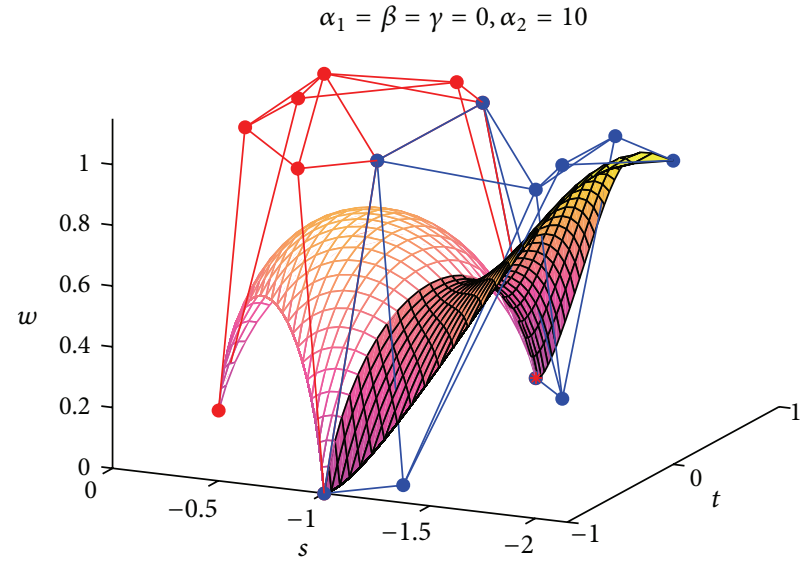

(b)

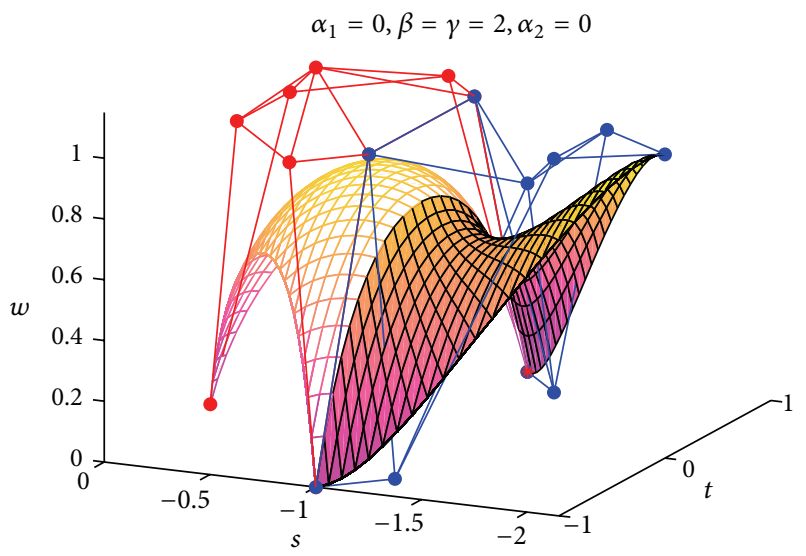

(d)

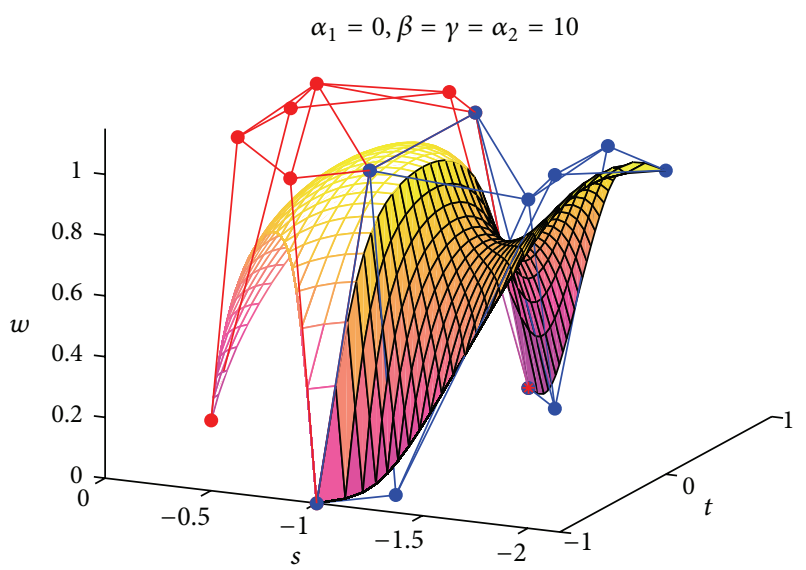

(f)

FIGURE 12: $G^{1}$ continuous surfaces with different shape parameters.

$$
\begin{aligned}
& \left.\frac{\partial R_{2}(t, s, 1-t-s)}{\partial t}\right|_{t=0} \\
& \quad=\frac{s[2+\beta+\beta(1-s)]}{[1+\beta(1-s)]^{2}}\left(Q_{1,2,0}-Q_{0,2,1}\right)
\end{aligned}
$$$$
\begin{aligned}
& +6 s(1-s)\left(Q_{1,1,1}-Q_{0,1,2}\right) \\
& +\frac{(1-s)[2+\gamma+\gamma s]}{[1+\gamma s]^{2}}\left(Q_{1,0,2}-Q_{0,0,3}\right) .
\end{aligned}
$$ 
The condition for smooth joining is that the vectors defined by (49), (50), and (51) should be coplanar for any $s \in[0,1]$; see [28]. It can be expressed as

$$
\begin{aligned}
& \left.\frac{\partial R_{2}(t, s, 1-t-s)}{\partial t}\right|_{t=0} \\
& =\xi \frac{d R_{1}(0, s, 1-s)}{d s}+\left.\zeta \frac{\partial R_{1}(t, s, 1-t-s)}{\partial t}\right|_{t=0},
\end{aligned}
$$

where both $\xi$ and $\zeta$ are constants. From these discussions, we can easily obtain a rule:

$$
\begin{aligned}
& Q_{1,2,0}-Q_{0,2,1}=\xi\left(P_{0,3,0}-P_{0,2,1}\right)+\zeta\left(P_{1,2,0}-P_{0,2,1}\right), \\
& Q_{1,1,1}-Q_{0,1,2}=\xi\left(P_{0,2,1}-P_{0,1,2}\right)+\zeta\left(P_{1,1,1}-P_{0,1,2}\right), \\
& Q_{1,0,2}-Q_{0,0,3}=\xi\left(P_{0,1,2}-P_{0,0,3}\right)+\zeta\left(P_{1,0,2}-P_{0,0,3}\right) .
\end{aligned}
$$

In summary, we conclude the following theorem.

Theorem 9. For $\alpha_{l}, \beta, \gamma \in[0,+\infty), l=1,2$, the surface connecting (46) with (47) is $G^{1}$ continuous, on the condition that (48) and (53) hold.

From Theorem 9, we can know that the conditions for smoothly joining two quartic rational triangular Said-Balllike patches are similar to the ones necessary for joining two triangular Bernstein-Bézier cubic patches; see [28]. However, we can adjust the shape of the obtained $G^{1}$ continuous surface conveniently by changing the tension shape parameters in the quartic rational triangular Said-Ball-like patch. Figure 12 shows the $G^{1}$ continuous smooth surfaces generated by the joining of two quartic rational triangular Said-Ball-like patches with different shape parameters. The parameters take values $\xi=1, \zeta=-1$.

\section{Conclusion}

The four quartic rational Said-Ball-like basis functions have the properties of partition of unity, nonnegative property, and linear independence and include the cubic Said-Ball basis functions as a special case. The new proposed $C^{1}$ quartic rational Hermite interpolation spline can be used to construct $C^{1}$ positivity- or monotonicity-preserving interpolation splines by constraining the shape parameters in the spline. The quartic rational Said-Ball-like basis with three-shape parameters over a triangular domain is a new construction for geometric design and computing, which has the properties of partition of unity, nonnegativity, and linear independence, and includes the classical cubic Said-Ball basis over a triangular domain as a special case. Without changing the control net, the shape of the obtained patch can be adjusted conveniently by using the shape parameters. Future work will concentrate on applying the new proposed basis to construct shape preserving interpolation spline surfaces.

\section{Conflict of Interests}

The authors declare that there is no conflict of interests regarding the publication of this paper.

\section{Acknowledgments}

We would like to thank the anonymous reviewers and the editor for their valuable comments for improvements. The research is supported by the National Natural Science Foundation of China (nos. 60970097, 61173119, and 11271376), Program for New Century Excellent Talents in University (no. NCET-13-0590), Hunan Province Science and Technology Project (no. 2014FJ2008), the Open Project Program of the State Key Lab of CAD\&CG (no. A1414) at Zhejiang University, Graduate Students Scientific Research Innovation Project of Hunan Province (no. CX2012B111), and Mathematics and Interdisciplinary Sciences Project, Central South University.

\section{References}

[1] A. A. Ball, "CONSURF. Part one: introduction of the conic lofting tile," Computer-Aided Design, vol. 6, no. 4, pp. 243-249, 1974.

[2] A. A. Ball, "CONSURF. Part two: description of the algorithms," Computer-Aided Design, vol. 7, no. 4, pp. 237-242, 1975.

[3] A. A. Ball, "CONSURF. Part 3: how the program is used," Computer-Aided Design, vol. 9, no. 1, pp. 9-12, 1977.

[4] H. B. Said, "Generalized ball curve and its recursive algorithm," ACM Transactions on Graphics, vol. 8, no. 4, pp. 360-371, 1989.

[5] T. N. T. Goodman and H. B. Said, "Shape preserving properties of the generalised Ball basis," Computer Aided Geometric Design, vol. 8, no. 2, pp. 115-121, 1991.

[6] S. Hu, G. Wang, and T. Jin, "Properties of two types of generalized Ball curves," CAD Computer Aided Design, vol. 28, no. 2, pp. 125-133, 1996.

[7] Q. Q. Hu and G. J. Wang, "Rational cubic/quartic Said-Ball conics," Applied Mathematics - A Journal of Chinese Universities, vol. 26, no. 2, pp. 198-212, 2011.

[8] T. N. T. Goodman and H. B. Said, "Properties of generalized Ball curves and surfaces," Computer-Aided Design, vol. 23, no. 8, pp. 554-560, 1991.

[9] S. Hu, G. Wang, and J. Sun, "A type of triangular ball surface and its properties," Journal of Computer Science and Technology, vol. 13, no. 1, pp. 63-72, 1998.

[10] J. Chen and G. J. Wang, "Construction of triangular DP surface and its application," Journal of Computational and Applied Mathematics, vol. 219, no. 1, pp. 312-326, 2008.

[11] Y. Zhu and X. Han, "A class of $\alpha \beta \gamma$-Bernstein-Bézier basis functions over triangular domain," Applied Mathematics and Computation, vol. 220, pp. 446-454, 2013.

[12] J. W. Schmidt and W. Heß, "Positive interpolation with rational quadratic splines," Computing, vol. 38, no. 3, pp. 261-267, 1987.

[13] M. Sakai and J. W. Schmidt, "Positive interpolation with rational splines," BIT: Numerical Mathematics, vol. 29, no. 1, pp. 140-147, 1989.

[14] M. Z. Hussain and M. Sarfraz, "Positivity-preserving interpolation of positive data by rational cubics," Journal of Computational and Applied Mathematics, vol. 218, no. 2, pp. 446-458, 2008.

[15] M. Sarfraz, M. Z. Hussain, and A. Nisar, "Positive data modeling using spline function," Applied Mathematics and Computation, vol. 216, no. 7, pp. 2036-2049, 2010. 
[16] F. N. Fritsch and R. E. Carlson, "Monotone piecewise cubic interpolation," SIAM Journal on Numerical Analysis, vol. 17, no. 2, pp. 238-246, 1980.

[17] C. Manni and P. Sablonnière, "Monotone interpolation of order 3 by $\mathrm{C}^{2}$ cubic splines," IMA Journal of Numerical Analysis, vol. 17, no. 2, pp. 305-320, 1997.

[18] M. Sarfraz, "A rational cubic spline for the visualization of monotonic data," Computers and Graphics, vol. 24, no. 4, pp. 509-516, 2000.

[19] M. Sarfraz, "A rational cubic spline for the visualization of monotonic data: an alternate approach," Computers and Graphics, vol. 27, no. 1, pp. 107-121, 2003.

[20] M. Z. Hussain and M. Sarfraz, "Monotone piecewise rational cubic interpolation," International Journal of Computer Mathematics, vol. 86, no. 3, pp. 423-430, 2009.

[21] B. I. Kvasov, "Monotone and convex interpolation by weighted quadratic splines," Advances in Computational Mathematics, vol. 40, no. 1, pp. 91-116, 2014.

[22] F. Ibraheem, M. Hussain, M. Z. Hussain, and A. A. Bhatti, "Positive data visualization using trigonometric function," Journal of Applied Mathematics, vol. 2012, Article ID 247120, 19 pages, 2012.

[23] U. Bashir and J. Md. Ali, "Data visualization using rational trigonometric spline," Journal of Applied Mathematics, vol. 2013, Article ID 531497, 10 pages, 2013.

[24] M. Z. Hussain, M. Hussain, and A. Waseem, "Shape-preserving trigonometric functions," Computational and Applied Mathematics, vol. 33, no. 22, pp. 411-431, 2014.

[25] X. A. Han, Y. C. Ma, and X. L. Huang, "The cubic trigonometric Bézier curve with two shape parameters," Applied Mathematics Letters, vol. 22, no. 2, pp. 226-231, 2009.

[26] M. Sarfraz, M. Z. Hussain, and M. Hussain, "Modeling rational spline for visualization of shaped data," Journal of Numerical Mathematics, vol. 21, no. 1, pp. 63-87, 2013.

[27] H. Akima, "A new method of interpolation and smoth curve fitting based on local procedures," Journal of Association for Computing Machinery, vol. 17, no. 4, pp. 598-602, 1970.

[28] D. Salomon and F. B. Schneider, The Computer Graphics Manual, Springer, New York, NY, USA, 2011. 


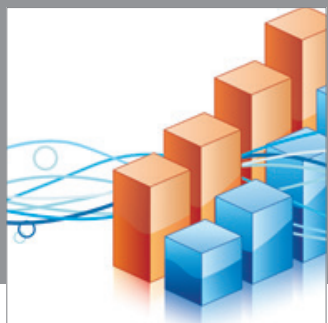

Advances in

Operations Research

mansans

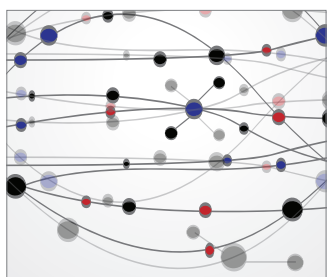

The Scientific World Journal
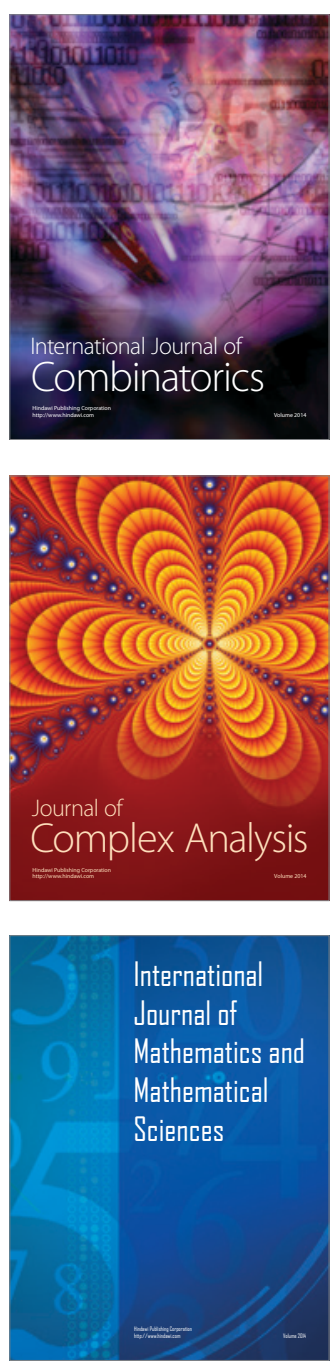
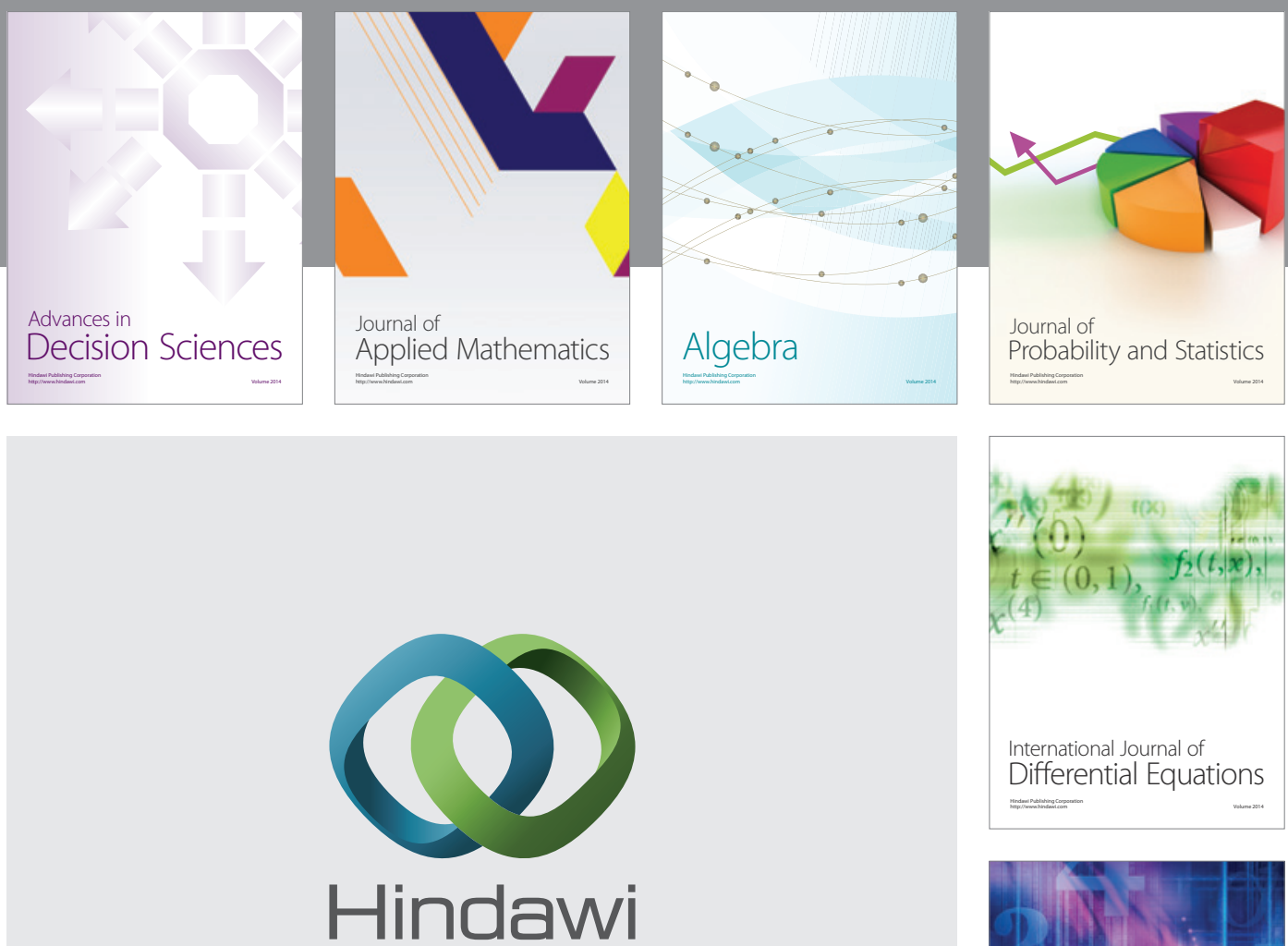

Submit your manuscripts at http://www.hindawi.com
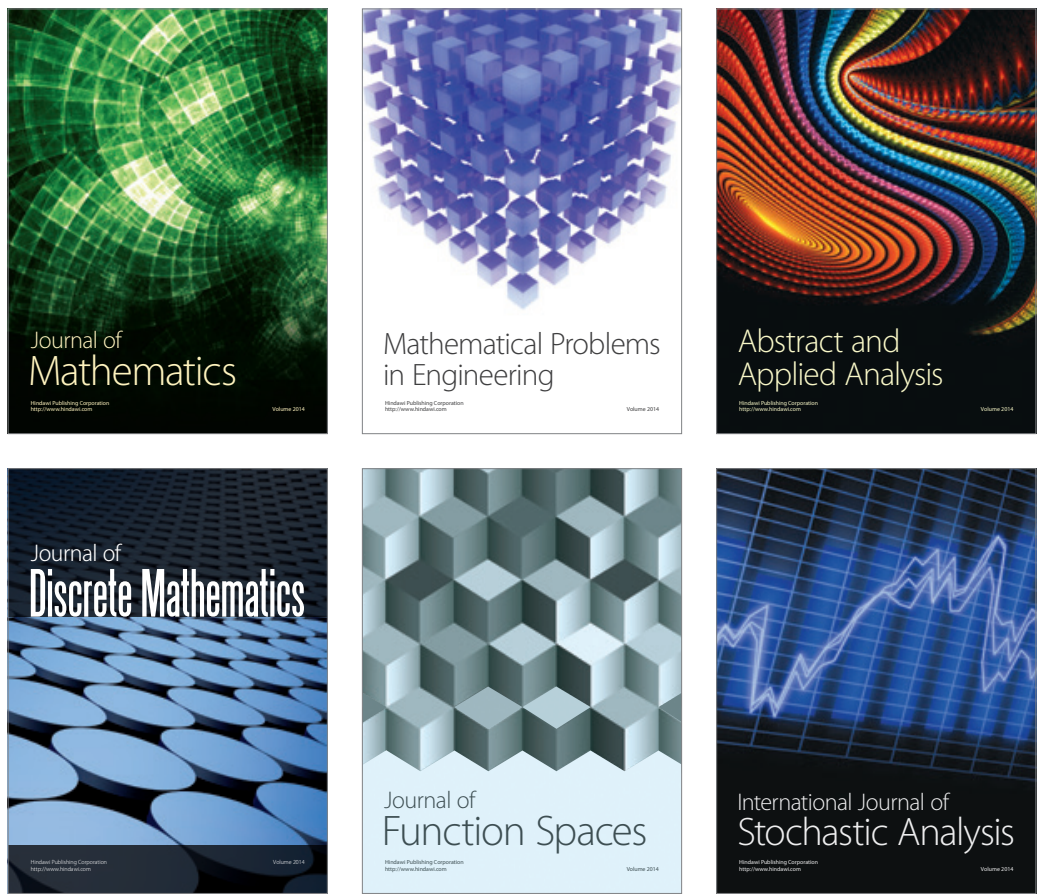

Journal of

Function Spaces

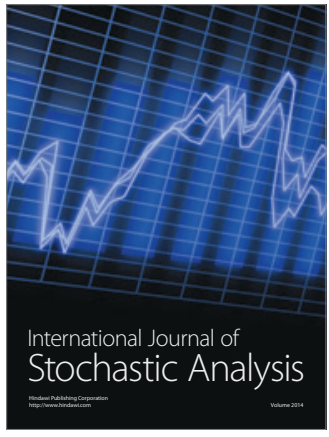

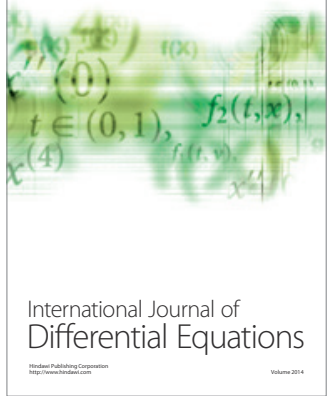
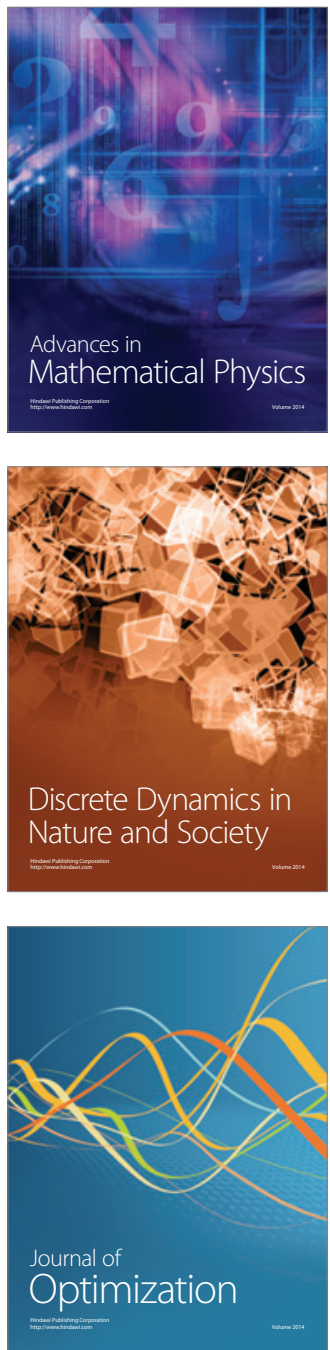